\title{
Watching Electrons Transfer from Metals to Insulators using Two Photon Photoemision
}

\author{
by \\ James E. Johns \\ Department of Chemistry \\ University of California, Berkeley \\ and \\ Chemical Sciences Division \\ Lawrence Berkeley National Laboratory \\ Berkeley, CA 94720
}

This work was supported by the Director, Office of Science, Office of Basic Energy

Sciences, of the U.S. Department of Energy under Contract No. DE-AC02-05CH11231. 


\section{DISCLAIMER}

This document was prepared as an account of work sponsored by the United States Government. While this document is believed to contain correct information, neither the United States Government nor any agency thereof, nor The Regents of the University of California, nor any of their employees, makes any warranty, express or implied, or assumes any legal responsibility for the accuracy, completeness, or usefulness of any information, apparatus, product, or process disclosed, or represents that its use would not infringe privately owned rights. Reference herein to any specific commercial product, process, or service by its trade name, trademark, manufacturer, or otherwise, does not necessarily constitute or imply its endorsement, recommendation, or favoring by the United States Government or any agency thereof, or The Regents of the University of California. The views and opinions of authors expressed herein do not necessarily state or reflect those of the United States Government or any agency thereof or The Regents of the University of California. 


\section{Watching Electrons Transfer from Metals to Insulators using Two Photon Photoemission}

By

James Johns

A dissertation submitted in partial satisfaction of the

requirements for the degree of

Doctor of Philosophy

in

Chemistry

in the

Graduate Division

of the

University of California, Berkeley

Committee in charge:

Professor Charles B. Harris, Chair

Professor Gabor Somorjai

Professor Mike Crommie

Spring 2010 


\section{Watching Electrons Transfer from Metals to Insulators using Two Photon Photoemission}

Copyright (C) 2010

By

James Johns 


\begin{abstract}
Watching Electrons Transfer from Metals to Insulators using Two Photon Photoemission
\end{abstract}

By

James Johns

Doctor of Philosophy in Chemistry

University of California, Berkeley

Professor Charles Harris, Chair

Ultrafast angle-resolved two photon photoemission was used to study the dynamics and interfacial band structure of ultrathin films adsorbed onto $\operatorname{Ag}(111)$. Studies focused on the image potential state (IPS) in each system as a probe for measuring changes in electronic behavior in differing environments.

The energetics and dynamics of the IPS at the toluene $/ \operatorname{Ag}(111)$ interface are strongly dependent upon coverage. For a single monolayer, the first IPS is bound by 0.81 $\mathrm{eV}$ below the vacuum level and has a lifetime of 50 femtoseconds (fs). Further adsorption of toluene creates islands of toluene with an exposed wetting layer underneath. The IPS is then split into two peaks, one corresponding to the islands and one corresponding to the monolayer. The wetting layer IPS shows the same dynamics as the monolayer, while the lifetime of the islands increases exponentially with increasing thickness. Furthermore, the island IPS transitions from delocalized to localized within $500 \mathrm{fs}$, and electrons with larger parallel momenta decay much faster. Attempts were made using a stochastic model to extract the rates of localization and intraband cooling at differing momenta.

In sexithiophene (6T) and dihexyl-sexithiophene (DH6T), the IPS was used as a probe to see if the nuclear motion of spectating side chains can interfere with molecular conduction. The energy and band mass of the IPS was measured for 6T and two geometries of DH6T on $\operatorname{Ag}(111)$. Electrons injected into the thicker coverages of DH6T grew exponentially heavier until they were completely localized by $230 \mathrm{fs}$, while those injected into $6 \mathrm{~T}$ remained nearly free electron like. Based off of lifetime arguments and the density of defects, the most likely cause for the mass enhancement of the IPS in this system is small polaron formation caused by coupling of the electron to vibrations of the alkyl substituents. The energetic relaxation of the molecular adsorbate was also measured to be $20 \mathrm{meV} / 100 \mathrm{fs}$ for the DH6T, and $0 \mathrm{meV} / 100 \mathrm{fs}$ for the $6 \mathrm{~T}$. This relaxation is consistent with the localization of the charge creating a barrier for it moving from one lattice site to a neighboring one.

Finally, the IPS was used to study the evolution of the surface band gap at the $\mathrm{Mg} / \mathrm{Ag}(111)$ interface. The $\mathrm{Mg}(0001)$ surface band gap lies $1.6 \mathrm{eV}$ below the Fermi level, and consequently shows no peak in the projected density of states for the IPS. A method for creating layer by layer growth of $\mathrm{Mg}$ on $\mathrm{Ag}(111)$ was determined using Auger Spectroscopy and low energy electron diffraction. By monitoring the decay of the 
intensity of the IPS versus coverage, it was determined that four layers of magnesium on $\operatorname{Ag}(111)$ is sufficient to completely eliminate the surface band gap 


\section{Contents}

\section{List of Figures}

\section{Introduction}

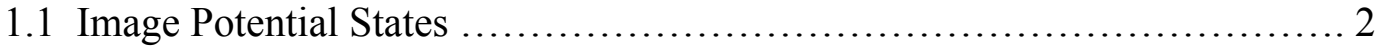

1.2 Two Photon Photoemission ........................................... 4

2. Experimental Apparatus 9

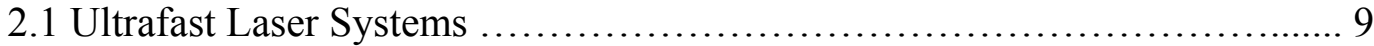

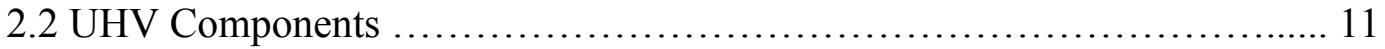

3. Toluene / Ag(111) 13

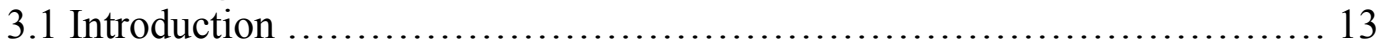

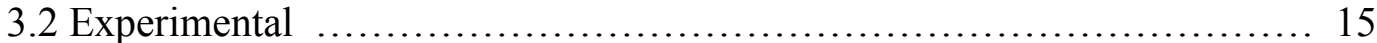

3.3 Results .................................................................. 15

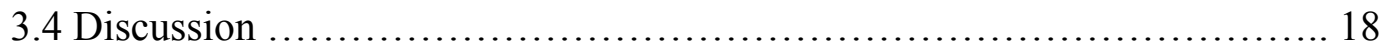

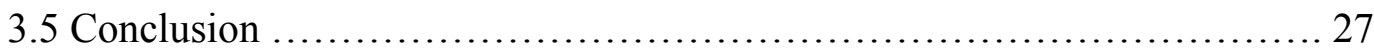

4. Charge Localization by alkyl side chains in Sexithiophene and DH6T 28

4.1 Introduction ........................................................ 28

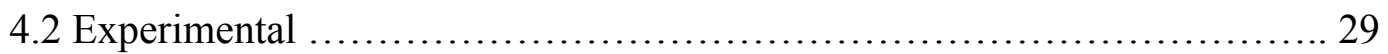

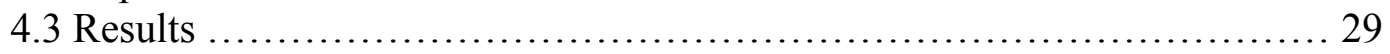

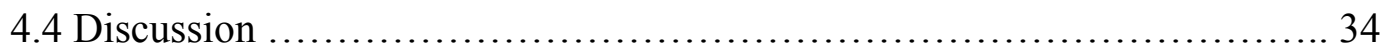

5. $\mathrm{Mg} / \operatorname{Ag}(111) \quad 37$

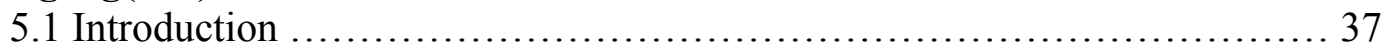

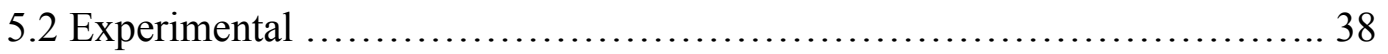

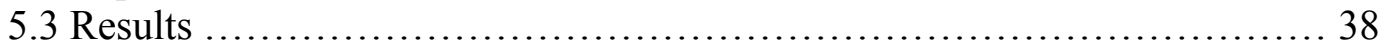

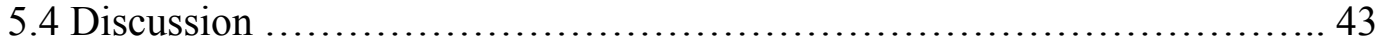

$\begin{array}{ll}\text { References } & 46\end{array}$ 


\section{List of Figures}

1.1 Dielectric Continuum Model Potential 3

1.2 Schematic of TPPE $\quad 5$

1.3 Angle Resolved TPPE 6

1.4 Different Processes following Photo-excitation 7

3.1 Adsorption Geometry of Toluene/ $\operatorname{Ag}(111) \quad 14$

3.2 Temperature Programmed Desorption of Toluene / $\operatorname{Ag}(111)$

3.3 Low Energy Electron Diffraction image of $\operatorname{Ag}(111)$ spots with Toluene 17 adsorbed

3.4 TPPE of 1 Monolayer of Toluene / $\operatorname{Ag}(111) \quad 19$

3.5 TPPE and Dynamics of Multilayers of Toluene / Ag(111) 21

3.6 Angle Resolved TPPE of Toluene Ag(111) showing Dynamic Localization 23

3.7 Fit of IPS Population vs Time and Angle using Friction Model for 26

Toluene/Ag(111)

3.8 Peak Width and Instrumental Resolution vs Time for Toluene / $\operatorname{Ag}(111) \quad 27$

4.1 Schematic of TPPE with adsorption geometries of 6T and DH6T 30

4.2 TPPE of 1ML 6T / Ag(111) 31

4.3 TPPE of Flat and Standing DH6T / Ag(111) 32

4.4 Energetic Relaxation of the $\mathrm{n}=1 \mathrm{IPS}$ for 6T and DH6T / $\mathrm{Ag}(111)$

4.5 Effective mass vs Time for 6T and DH6T / $\mathrm{Ag}(111)$

5.1 Auger Spectrum of $\mathrm{Mg} / \mathrm{Ag}(111) \quad 39$

5.2 Auger Dosing Survey $\mathrm{Mg} / \mathrm{Ag}(111)$ at $150 \mathrm{~K} \quad 40$

5.3 Auger Dosing Survey $\mathrm{Mg} / \mathrm{Ag}(111)$ at Room Temperature 41

5.4 LEED of $\mathrm{Mg} / \mathrm{Ag}(111) \quad 42$

5.5 TPPE of $\mathrm{Mg} / \mathrm{Ag}(111) \quad 43$ 


\section{Acknowledgements}

First and foremost, I would like to thank my beautiful and brilliant wife. Without her support, I neither would have made it through the bad times, nor enjoyed the good times nearly so much. Secondly, I need to express my gratitude to my advisor Charles Harris, for giving me the intellectual freedom and support to explore the projects which captivated my interests. And thirdly, I need to thank Vijaya Narasimhan, our group mother and administrative assistant. Without her helpful reminders and persuasive phone calls, I know there would have been several semesters in which I was neither registered nor paid.

Intellectually I must acknowledge the role of the people who came before me in the group and who influenced the way that I think about electron dynamics at surfaces. Steve Shipman had a passionate curiosity about all things scientific, and was a great motivator for reading the highest volume of science possible. Sean Garret-Roe lent the lab an intellectual rigor and authority that each of us who follows must struggle to attain. Matt Strader and Aram Yang served as personal mentors, and taught me the ins and outs of the experimental nightmare that is two photon photoemission. Without their help, I never would have learned how to make a truck full of giraffes all duck their heads at the same time. And of course, Eric Muller forced me to rethink everything I thought I knew in order to teach him without embarrassing myself. Also, without his competency in the student shop, I would still be waiting on parts to take data.

I would like to thank the people in the lab that I never worked with directly for helping to make the lab a fun and creative environment. I would also like to express my appreciation to Pradeep Nair and David Kavulak. Without such good friends, grad school would have been a much bleaker time. And finally, I would like to thank my parents Jim and Terry Johns for their love and intellectual nurturing.

This work was supported by the Director, Office of Energy Research, Office of Basic Energy Sciences, Chemical Sciences Division of the U.S. Department of Energy, under Contract No. DE-AC02-05CH11231. 


\section{Chapter 1}

\section{Introduction}

As the scientific community continues a trend towards miniaturization, new and interesting phenomena have been discovered. The blossoming field of nanoscience has examined the peculiar effects of what happens when macroscopic systems are shrunk to sizes where the wave-like quantum nature of matter begins to dominate its behavior. When electrons are confined to a length scale below $\sim 10 \mathrm{~nm}$, roughly the wavelength of a room temperature electron, its behavior shifts, and it can exhibit some rather peculiar properties. These properties can also have a strong dependence on the dimensionality of the system. For example quantum dots, model 0 dimensional systems, can be turned into artificial atoms. Quantum confinement along the axis of carbon nanotubes, a one dimensional system, is responsible for its unique electron transport properties. But far and away the most ubiquitous confinement to nanometer length scales occurs at surfaces.

Although surfaces have been actively studied for decades for their unique catalytic and bonding properties, it is not until recent technological advances in femtosecond lasers (lasers that operate with pulses as short as $10^{-14}$ seconds) allowed for direct examination of how electrons behave over time. Electrons at conducting surfaces tend to have a very short lifetime, and require such ultrafast experimental probes in order to investigate what they are doing. One of the most controllable classes of surfaces that has been studied is a metal surface covered by an atomically thin molecular layer. This system is of interest both for the diversity of environments on both sides of the interface, and because it is a system of technological relevance. For example, charges must always be transferred across the metal/molecular interface when extracting charges out of an organic solar cell or injecting them into an organic light emitting diode (OLED).

Electron dynamics in ultrathin films of molecular adsorbates have been studied in order to better understand a variety of processes including two dimensional solvation, modeling electrochemical interfaces, and interfacial electron transfer. One of the underpinning motivations in all of these works is attempting to understand how the behavior of a single electron changes when it crosses the boundary between these two fundamentally different environments. Valence electrons in non-transition row metals are well described as highly delocalized quasiparticles, whereas electrons in molecules are in highly localized, highly directional chemical bonds. Even for films of crystalline organic semiconductors, their electronic properties are determined first and foremost by the chemical bonding of the single molecule, and further delocalization can be treated perturbatively. 


\subsection{Image Potential States}

When a charge is injected from a metal into a dielectric material, it induces a polarization both in the dielectric and in the metal. This potential provides an attractive force which attempts to pull the charge back in to the metal surface. The potential is known as the image potential, because the classical analogue of this problem can be solved by using the method of images ${ }^{1}$. The quantum mechanical origins of the potential at the single electron level are due to an exchange correlation interaction between an electron at the interface and electrons of the same spin in the metal near the surface ${ }^{2-4}$. Classically, the potential takes the form of Equation 1.1

$$
V(z) \propto-\frac{1}{4(z)}
$$

where $\mathrm{z}$ is the distance from the surface. This potential acts upon the electron perpendicular to the surface, but does not have any effect parallel to the surface.

Electrons bound by the image potential (IPS) were first observed at the surface of liquid helium in 1973 Grimes and Brown ${ }^{5}$. Since then, they have been experimentally investigated on many different surfaces including the noble metals ${ }^{6,7}$, transition metals ${ }^{2,8-}$ ${ }^{10}$, silicon ${ }^{11}$, graphene ${ }^{12}$, and carbon nanotubes ${ }^{13}$. For metallic systems with a surface band gap between the Fermi level and the vacuum level, image potential creates a Rydberg series of bound electronic states. The energy of the image potential states (IPS) takes a particularly simple form given by Equation 1.2

$$
E_{n}=-\frac{0.85 e V}{(n+a)^{2}}+V_{0}
$$

where $\mathrm{n}$ is the principle quantum number, $\mathrm{a}$ is a quantum defect parameter, and $\mathrm{V}_{0}$ is vacuum energy ${ }^{14,15}$. The quantum defect parameter is usually $<0.1$ and depends on the relative position of the IPS to the onset of the conduction band. For the $\operatorname{Ag}(111)$ surface, the binding energy, or energy below the vacuum level, has been experimentally measured at $-0.77 \mathrm{eV}$ and $-0.23 \mathrm{eV}$ for the $\mathrm{n}=1$ and $\mathrm{n}=2$ states respectively. The lifetime of the IPS is determined by its overlap with the bulk of the metal ${ }^{16}$. Image states at the metal surface typically exist for 0-30 fs, while high quantum number states which are further from the surface can exist for up to picoseconds $2,8,16,17$. The average distance from the metal surface for the $n=1$ IPS is only $3 \AA$. The proximity to the surface makes it sensitive to the presence of adsorbates and an ideal probe to see how electrons bridge the divide from metallic to non metallic environments ${ }^{18}$.

The question of how to modify the image potential in a quantitative fashion due to a dielectric film was first addressed by Cole in $1970^{19,20}$. Cole solved Maxwell's equation for the potential felt by a charge outside the surface of a metal with a uniform film characterized only by its dielectric constant and its thickness. A modified version accounting for the electron affinity (here defined as the energy of the conduction band relative to the vacuum level) of the material was first applied to image potential electrons in 1996 by Lingle et al. ${ }^{21}$ The resultant screened image potential is shown in Figure 1.1. This model takes the low frequency dielectric constant, electron affinity, and film thickness as inputs. The resultant potential can be inserted into the time independent Schrödinger equation which must be solved numerically. The lifetime of these states are 
determined by the penetration of the image potential wave functions into metal and the known lifetime of electrons in the metal at that energy ${ }^{22}$. Adsorbates with negative electron affinities should from a barrier that pushes the electron away from the surface, increasing its lifetime. This model has been successfully used to explain the IPS energetic positioning and lifetime for several adsorbate systems including noble gasses ${ }^{22}$, ${ }^{23}$, linear acenes ${ }^{24}$, and alkanes ${ }^{21,25}$.

\section{Dielectric Continuum Model Potential}

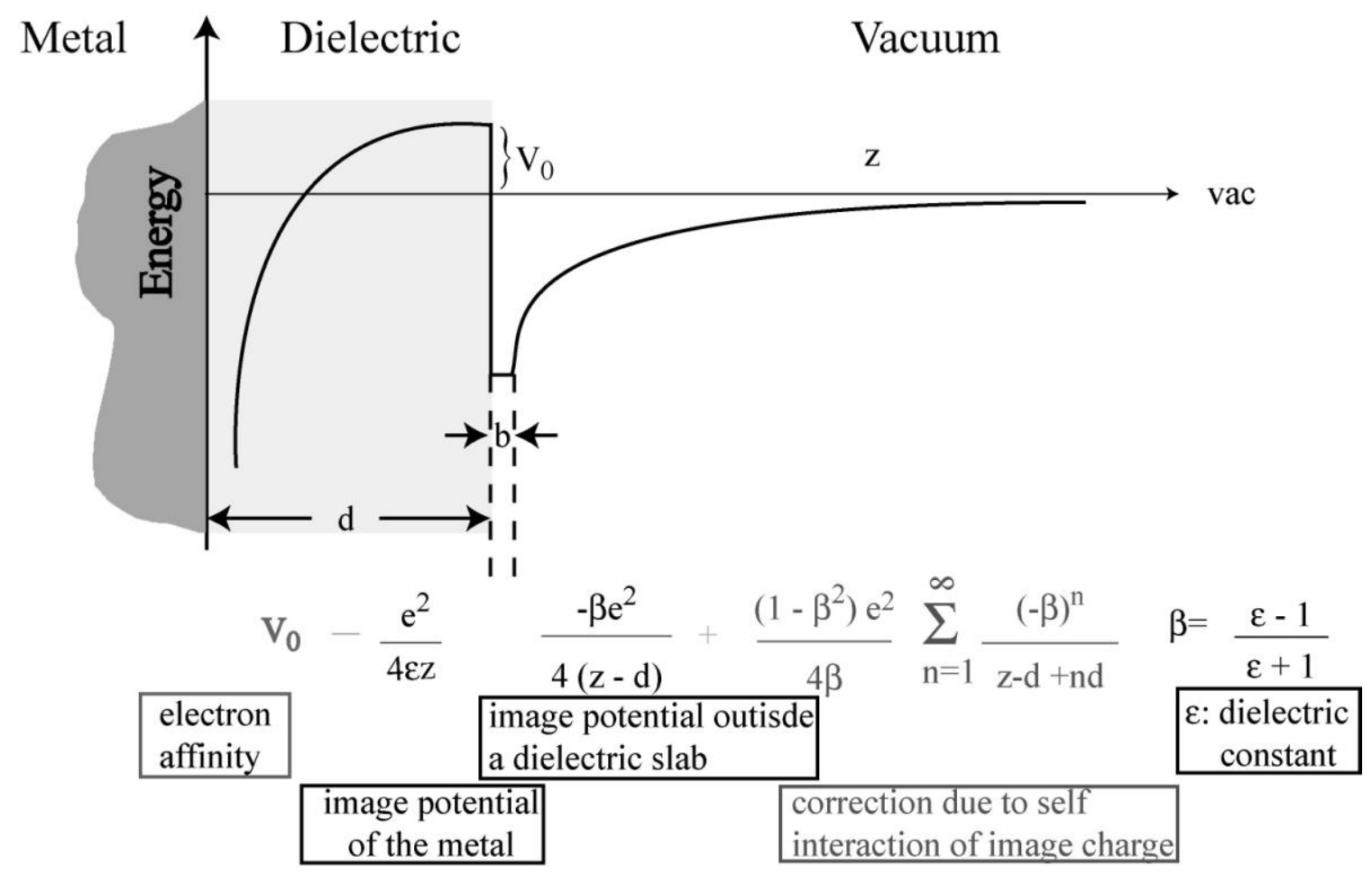

Figure 1.1 Dielectric Continuum Model for the image potential as solved by Cole

This model, however, also has several inadequacies. The DCM is a structureless model. As a result, it cannot account for materials with similar physical properties but with differing emergent behavior as a result of differing morphological or crystalline properties. For example, the dynamics of IPS electrons have been measured for small aromatics physisorbed to $\mathrm{Ag}(111)$. Molecules such as benzene, para-, ortho-, and metaxylenes all have very similar electron affinity levels and nearly identical dielectric constants as shown in Table $1.1^{26-28}$.

The dielectric continuum model can offer no insight into why the image potential states at these very similar molecular interfaces behave so differently. All three of these molecules have a measured positive electron affinity, which should lead to the IPS being at the metal/molecule interface with sub 100 fs decays. Furthermore, with the exception of benzene, the dynamics of the IPS in these states are not simple exponential decays as the model would propose. The DCM becomes even worse as the electron affinity increases. For organic semiconductors, such as perylenes, fullerenes, and pentacene, the 
electron affinity level is in excess of $2 \mathrm{eV}^{7,29-33}$. Nevertheless, each of these systems have experimentally verified image potential states located within $0.9 \mathrm{eV}$ of the vacuum level, rather than converging to the electron affinity level as the model would predict. Thus, although it captures some of the basic physical elements for many systems, it remains insufficient as a general model for the interpretation of IPS with adsorbates, and new methods are required.

\begin{tabular}{|l|l|l|l|}
\hline Molecule & Electron Affinity & $\begin{array}{l}\text { Dielectric } \\
\text { Constant }\end{array}$ & $\begin{array}{l}\text { Trilayer } \\
\text { Exponential } \\
\text { Decay Time (fs) }\end{array}$ \\
\hline Benzene & $0.5 \mathrm{eV}$ & 2.3 & 50 \\
\hline o-Xylene & $0.4 \mathrm{eV}$ & 2.6 & 200 \\
\hline $\mathrm{m}-$ Xylene & $0.38 \mathrm{eV}$ & 2.4 & 400 \\
\hline p-Xylene & $0.5 \mathrm{eV}$ & 2.3 & 1000 \\
\hline
\end{tabular}

Table 1.1 Input properties for the DCM and experimental lifetimes. Despite the similarities of their dielectric properties, these molecules have different lifetimes by a factor of $\mathbf{2 0}$ for the same thickness.

Unfortunately, there exist very few other methods for predicting the energetics and dynamics of the IPS at a metal surface with complex organic adsorbates. For a variety of reasons, density functional theory (DFT) underestimates the electron spill out of metallic surfaces due to the vanishing band gap in the bulk and the superimposed surface band gap. It also fails to accurately yield the correct functional form of the image potential. Progress has been made (and previously described therein) in the creation of one dimensional potentials which effectively match the work function, image potential, and surface state to known experimental values ${ }^{34}$. Employing the GW approximation to LDA-DFT results in the accurate prediction of electronic energies and lifetimes, as well as breaking down these lifetimes into contributions from phonon contributions, electronhole contributions, and electron-electron contributions.

This task, however, is inherently difficult and computationally expensive. To date, the most complex system described in this level of detail is a monolayer of cesium over silver adlayers deposited on copper ${ }^{35,36}$. If, however, we consider a relatively simple system (from a chemist's perspective) such as linear alkanes deposited on a metal, the DFT is computationally far too expensive. If each unit cell contains only a single alkane, this still requires several metal atoms per unit cell, each of which drastically increases the computational time required. The computational formulism and power does not yet exist to study metal induced excited states at surfaces in these systems. Therefore more experimental evidence of how these interfacial electrons behave is needed in order to make better predictive models

\subsection{Two Photon Photoemission}


The method of choice for making experimental observations of IPS electrons is two photon photo-emission (TPPE). TPPE is an ultrafast pump-probe technique used to investigate excited state surface electrons, typically of metals or adlayers supported on metal substrates. A laser pump pulse with energy $h v_{1}$ excites an electron from below the Fermi level of the metal to an intermediate state. After some waiting time, $\tau$, a probe pulse with energy $h v_{2}$ photo-emits excited electrons. The kinetic energy of these electrons is then measured either by measuring flight time to a detector or using an electron energy analyzer. By subtracting the energy of the probe pulse from the measured kinetic energy spectrum, one can determine the absolute energy level of electronic states relative to the Fermi and vacuum levels. By changing the pump-probe delay time, changes in the energy of electronic states can be monitored. This is outlined in Figure 1.2.

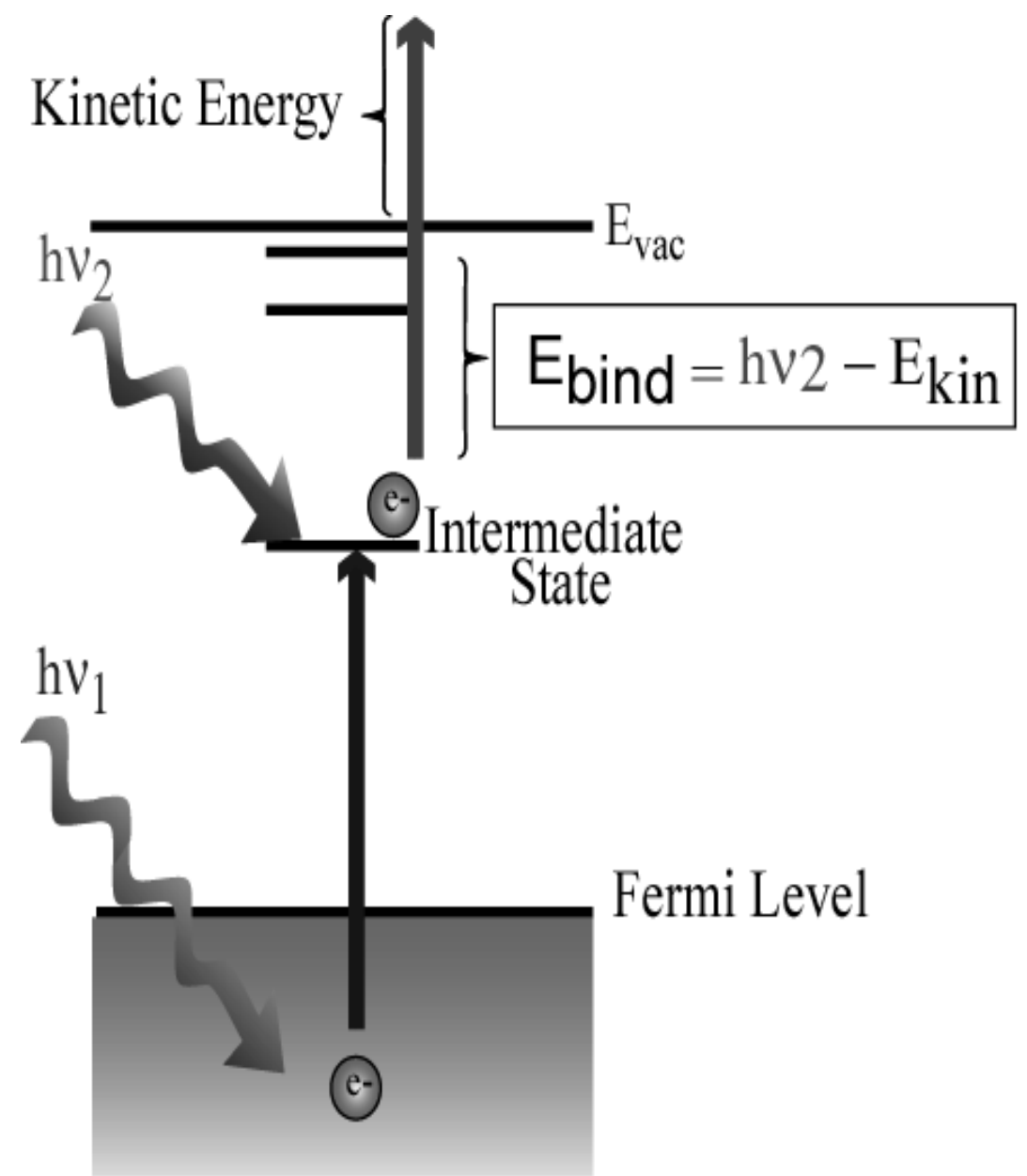

Figure 1.2 Diagram of the two photon photoemission process

The ability to time resolve photoemission of excited states is further improved by the ability to momentum resolve states as shown in Figure 1.3. At a crystal surface, quasiparticles bound in the $\mathrm{z}$ direction (surface normal) still have a two dimensional band structure, $\mathrm{E}\left(\mathrm{k}_{\mathrm{x}}, \mathrm{k}_{\mathrm{y}}\right)$. The momentum of an electron parallel to a well defined surface has 
been shown to be preserved during photoemission. Electrons of different momenta parallel to the surface are therefore photo-emitted at different angles to the surface normal related by the Equation 1.3:

$$
k_{x}=\frac{\sqrt{2 m_{e} E_{\text {Kinetic }}}}{\hbar} \sin (\theta)
$$

where $\mathrm{k}_{\mathrm{x}}$ is the momentum wavevector, me is the mass of an electron, $\mathrm{E}_{\text {kinetic }}$ is the kinetic energy, and $\theta$ is the angle of the photo-emitted electron relative to the surface normal

A)
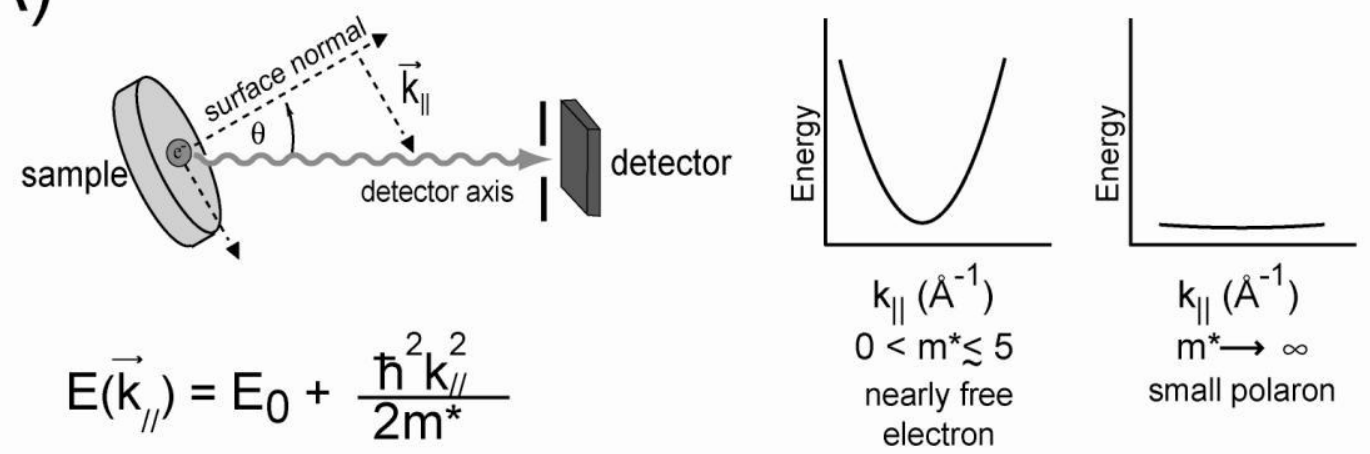

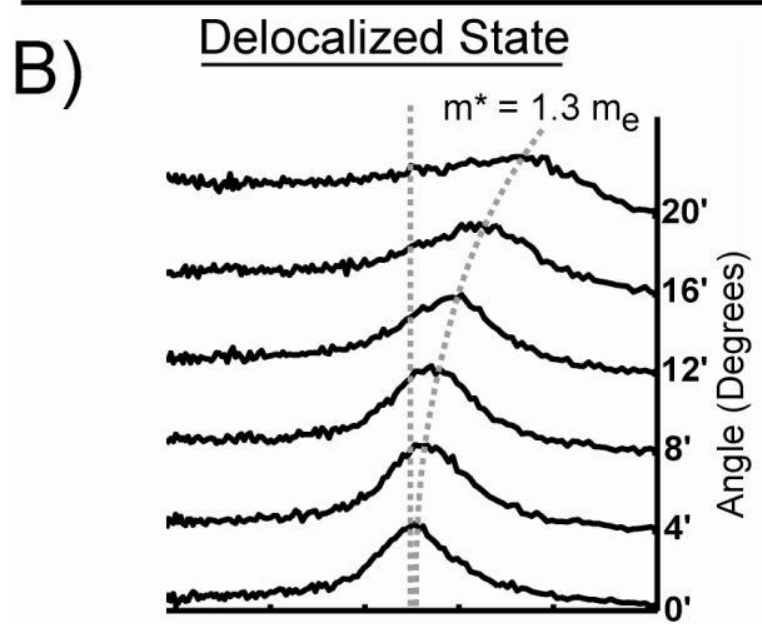

Binding Energy (eV)

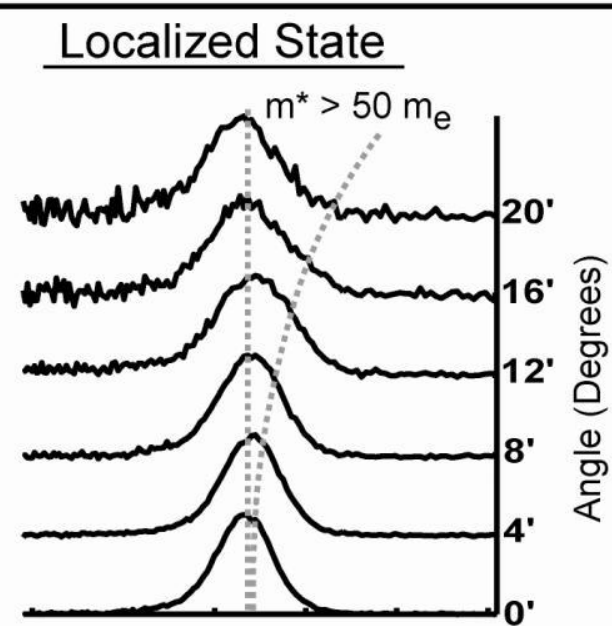

Binding Energy (eV)

Figure 1.3 A) Diagram showing the ability of TPPE to measure differing parallel momenta by adjusting the angle. Only electrons with a specific parallel momentum as a function of kinetic energy will make it to the detector at any given angle. B) Experimental example of a localized and a delocalized state, taken on the same system ( $3 \mathrm{ML}$ of toluene/ $\mathrm{Ag}(111))$ at different times.

vector. Angle resolved spectra can be taken by either varying the angle of the sample with respect to a fixed detector with a limited acceptance angle, or by using an array style detector. For spectra taken where the momentum in the $\mathrm{x}$ and $\mathrm{y}$ directions are the same, or laser spot size averages over many domains oriented in different directions, the momentum is often referred to as $\mathrm{k}_{/ /}$since a more specific direction is often 
experimentally impossible to define. From the measured surface band structure, we can determine the effective mass $\left(\mathrm{m}^{*}\right)$ using Equation 1.4

$$
m^{*}=\hbar^{2}\left[\frac{\partial^{2} E}{\partial k_{/ /}^{2}}\right]^{-1}
$$

For metal derived surface states, such as bare image potential states (IPS) or Shockley surface states, the effective mass is approximately 1 , and usually less than $5^{6}$. For systems in which an electron is highly localized, either because of strong material interactions or a lack of crystallinity, the energy of the state does not depend on the momentum, ie $\mathrm{k}$ is not a good quantum number, and the effective mass approaches infinity. Example processes that can cause this are energetically disordered landscapes ${ }^{37}$, charge trapping at defects ${ }^{38}$, or small polaron formation ${ }^{39}$.

\section{Interband Transition}

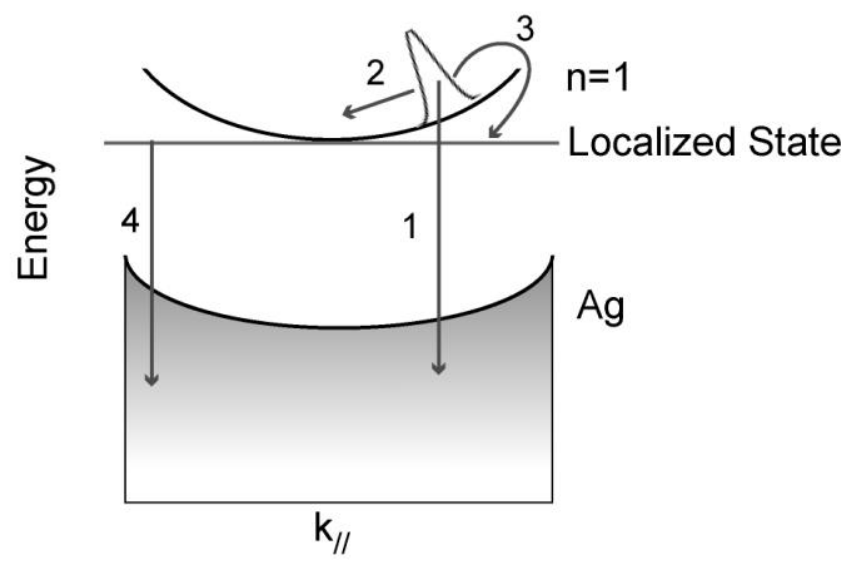

2. Intraband Relaxation

3. Localization

4. Interband Transition from the Localized State

Figure 1.4 A schematic illustrating different processes observable by TPPE which can happen to excited electron

Finally, neither the energy nor the band curvature, nor the relative intensity of a given k-state of an excited IPS electron needs be a constant value. This is sketched out in figure 1.4. Once an electron is excited across the surface boundary, it can interact with the adsorbed layer. Molecules with a strong permanent dipole may rotate in order to have the positive portion of the dipole point towards the electron. This is the two dimensional equivalent of solvation as studied by many other ultrafast optical spectroscopists in the liquid phase ${ }^{40,41}$. Electrons in high momenta states can relax down to lower states by making intraband transitions. There can be electron transfer events in which an excited electron hops from one band to another. The excited electrons can scatter off of defects in the material or molecular vibrations and transition from delocalized to localized states. All of these dynamic processes can be studied by time and angle resolved TPPE in order 
to gain information about how electrons behave at the interface between metals and molecules. 


\section{Chapter 2}

\section{Experimental Apparatus}

The machinery required for taking time and angle resolved two photon photoemission can be broken into four primary parts: ultrafast lasers, ultrahigh vacuum equipment, electron detection equipment, and sample preparation equipment. The original design of the system UHV systems is explained in more detail in the thesis of Walt Merry ${ }^{42}$. The photoelectron detection system is described most thoroughly in Jason McNeil's thesis ${ }^{43}$. The Knudsen cell used in these experiments was built by Sean Garrett-Roe and is written about in his thesis ${ }^{28}$. Below is a description of the TPPE spectrometer as used in the experiments in this thesis with an emphasis given to changes in hardware and details which could prove salient in further upgrades to the laser system.

\subsection{Ultrafast Laser System}

The tunable ultrafast pulses used in these experiments were generated by commercially available Coherent Inc ${ }^{\circledR}$ lasers. An array of solid state LEDs are coupled by a fiber optic to an $\mathrm{Nd}: \mathrm{YVO}_{4}$ continuous wave lasing cavity with a lithium triborate doubling crystal in the Verdi ${ }^{\mathrm{TM}} 18 \mathrm{~W}$ laser which produces $18 \mathrm{~W}$ of $532 \mathrm{~nm}$ light. This pump laser replaced two separate 5 and $10 \mathrm{~W}$ Verdis ${ }^{\mathrm{TM}}$ which were suffering internal damage in the laser head. Using a beam splitter, $6 \mathrm{~W}$ are directed into a Ti:Sapphire oscillator (Mira 900F) which produces a mode locked train of pulses centered at $800 \mathrm{~nm}$ at a rate of $76 \mathrm{MHz}$. The oscillator is easily tuned between 750 and $850 \mathrm{~nm}$ via a birefringent filter in the lasing cavity. Adjusting the wavelength also required changing the amount of glass in the oscillator's prism compressor in order to maintain mode locking. These pulses were then regeneratively amplified in a REGA9000 ${ }^{\mathrm{TM}}$ at $225 \mathrm{kHz}$. The resultant output was 1.2 to $1.5 \mathrm{~W} 800 \mathrm{~nm}$ light in $150-200$ fs pulses at $225 \mathrm{kHz}$.

In order to increase the efficiency of downstream nonlinear optical components, attempts were made to shorten the duration of the pulses from the amplifier by increasing the bandwidth. Typical bandwidth of pulses from the oscillator are 10-12 nm FWHM, and Ti:Sapph oscillators have been shown to be able to produce much broader pulses with a spectral FWHM of $\sim 50 \mathrm{~nm}$. At first, it was assumed that the birefringent tuning crystal in the oscillator was the limiting factor governing maximum bandwidth in the system. However, it was later determined that the limiting factor was the length of the Ti:Sapphire lasing crystal. In early models of the REGA ${ }^{\mathrm{TM}}$ and Mira ${ }^{\mathrm{TM}}$ such as those used in our lab, the oscillator and amplifier crystals are between 2.5 and $3 \mathrm{~cm}$ in length rather than the single centimeter crystals used in later models. This leads to extra group 
velocity mismatch beyond the second order dispersion which can be corrected for by the prism pair. In order to achieve more broadband pulses, new crystals and mounts would need to be installed in the oscillator and amplifier.

Post amplification, the beam then enters a collinear double pass optical parametric amplifier (OPA) which generates tunable laser pulses from $475 \mathrm{~nm}$ to $720 \mathrm{~nm}$, with a temporal duration of 60 to 120 fs following compression in a prism pair based compressor. Typical average powers are between 20 and $50 \mathrm{~mW}$. This light is then frequency doubled in a $0.7 \mathrm{~mm}$ thick type I BBO crystal. The resultant ultraviolet light is separated from the visible via a dichroic mirror. The two beams are adjustably delayed by a translation stage before being collinearly recombined and directed at the sample. The cross correlation of the two beams was determined at the start of every experiment by taking dynamic scans of the clean $\mathrm{Ag}(111)$ surface. Intensity of the direct photoemission of the non-resonant background for silver was fit to a Gaussian peak and determines the instrument response function, typically 90 to $130 \mathrm{fs}$ depending on the wavelength of the visible pulse.

This approach is sufficient for examining electronic states which are energetically closer to the vacuum level than the Fermi level. However, it is insufficient for studying on molecular excitations because of work function limitations, and because the two pulses are constrained to one tunable pulse and its second harmonic. In order to observe TPPE signals, it is imperative that the energy of the ultraviolet pulse be kept below the work function of the material being studied. For many organic dye molecules used for light harvesting such as $\mathrm{N}$-thiophenes, and fluorenes, the work function ranges from 3.4 to $4.0 \mathrm{eV}$. The peak optical absorption for these materials, however, are often over $2 \mathrm{eV}$. Conversely, for many systems such as the phthalocynines, the work function is $\sim 4$ to 4.3 $\mathrm{eV}$, but the peak absorption occurs as low as $1.7 \mathrm{eV}$. If the surface donates charge into the organic semiconductor upon adsorption, the gap between the Fermi level and the molecular LUMO can be lowered to a value below the work function minus the energy of the ultraviolet photon. This results in optical pumping at the surface but no photoelectrons that can be detected. In order to investigate molecular excitation in thin films, a second tunable pulse is necessary.

Thus, once the new $18 \mathrm{~W}$ Verdi was installed, I began work constructing a noncollinear optical parametric amplifier (NOPA). The majority of ultrafast nonlinear optical experiments use $1 \mathrm{kHz}$ regenerative amplifiers which are optically pumped either by flash lamps or Q-switched lasers. This results in significantly higher peak energies and electric fields per pulse than the $225 \mathrm{kHz}$ system used in this experiment. The advantage of a higher peak power is that many groups have used such an amplifier to employ three or more tunable OPAs through judicious use of available photons. However, only a single other research group has been found which converts the output of a high rep rate amplifier into two fully tunable pulses ${ }^{44}$.

Using a 50\% beam splitter, the output of the amplifier was split into two beams. One of these beams was then frequency doubled to provide a $400 \mathrm{~nm}$ pump pulse, and the residual $800 \mathrm{~nm}$ was separated via a dichroic mirror. The $800 \mathrm{~nm}$ was then focused into a sapphire window to generate a super continuum, which was focused into a $1 \mathrm{~mm}$ type 1 $\mathrm{BBO}$ crystal. The $400 \mathrm{~nm}$ was directed through a delay stage to a curved mirror, and focused into the $\mathrm{BBO}$ at an angle of $\sim 5^{\circ}$. By varying the delay time of the ultraviolet 
pulse, overlap of the two beams, and the angle of the BBO, broadband phase matching was established with a FWHM of $35 \mathrm{~nm}$ centered at $600 \mathrm{~nm}$. This served as proof of principle that the output of the amplifier was sufficient to operate two OPAs. The crystals used to build this secondary NOPA, however, were visually flawed, and resulted in a highly distorted output. Therefore, the NOPA was dismantled and new crystals were ordered.

\subsection{UHV Components}

All TPPE experiments were performed on a $1.5 \mathrm{~cm} \mathrm{Ag(111)} \mathrm{single} \mathrm{crystal.} \mathrm{This}$ crystal was ordered from MaTecK $\mathrm{GmbH}$ as a $99.999 \%$ pure crystal. In order to maintain a surface free from contaminants, experiments carried out in ultrahigh vacuum at pressures under $4 \times 10^{-10}$ torr. Base pressure in this chamber is $8 \times 10^{-11}$ torr when pumped by both a cryogenic braid and an ion pump. The crystal is mechanically attached to a Chromel-Alumel type thermocouple, and is otherwise electrically isolated from the underlying heater and the chamber by insulating sapphire beads. During the collection of TPPE data, the negative lead of the thermocouple was connected to ground outside of the chamber in order to electrically ground the sample. The crystal base plate is mounted on a 5 -axis manipulator arm which allows for $\mathrm{x}_{-}^{-}, \mathrm{y}-$, and $\mathrm{z}$ - positioning to $\pm 0.1 \mathrm{~mm}$, and angular orientation to \pm 1 degree. The sample can be cryogenically cooled by a Janis ${ }^{\circledR}$ ST-400 cryostat or resistively heated from $50 \mathrm{~K}$ to $900 \mathrm{~K}$.

Sample preparation in UHV is initiated by cleaning the silver crystal. For most adsorbates, sputtering by $500 \mathrm{eV} \mathrm{Ar}^{+}$ions at $500 \mathrm{~K}$ and annealing at $725 \mathrm{~K}$ is sufficient to remove them from the surface and restore a clean $\operatorname{Ag}(111)$ surface. For one experiment, however, this was insufficient. Magnesium is known to form a surface alloy with the $\operatorname{Ag}(111)$ surface if it is annealed above $450 \mathrm{~K}$. Sputtering and annealing the crystal was insufficient to remove this, and ex situ mechanical polishing was required using silica colloids. Sample cleanliness was checked daily by using TPPE to examine the position and dynamics of the bulk sp band transition, Shockley surface state, and n=1 IPS.

Surface structure was checked periodically using low energy electron diffraction from an Omicron ${ }^{\circledR}$ SPECTALEED unit.

Molecules can be delivered to the crystal surface either by introducing them into the entire chamber through a precision variable leak valve or by molecular beam epitaxy. Liquids or gases with a vapor pressure greater than 0.5 torr at room temperature are suitable for dosing via the leak valve. This is accomplished by filling a small antechamber with vapor, and slowly opening the valve into the main chamber. Pressure in the chamber is monitored during dosing by an ion gauge, and coverage at the sample can be estimated from the exposure in Langmuirs. This method has the distinct disadvantage of coating everything else in the chamber including filaments and electron optics with the molecule being dosed, but is easiest way to dose small molecules.

Molecules or atoms which do not have appreciable vapor pressure at room temperature can be dosed via a molecular beam. The material is inserted into a ceramic crucible, which is then restively heated in an attached high vacuum chamber until there is sufficient vapor pressure. The gate valve which separates the two chambers is then 
opened, allowing for mass transport. A shutter and a mask with a $1 \mathrm{~cm}$ hole prevent unnecessary contamination of the main chamber during dosing. The crucible to surface distance is quite long ( $34 \mathrm{~cm}$, adjustable to $25 \mathrm{~cm}$ ) which results in a relatively uniform flux at the $1.5 \mathrm{~cm}$ wide silver crucible.

Two photon photoemission spectra are taken by time correlated single electron counting. A portion of the laser pulse is picked off by a photodiode and the resultant electrical signal is used as a start time. Low energy photo-electrons from the sample travel a distance of $13 \mathrm{~cm}$ inside a shielded flight tube and are amplified by a pair of multichannel plates. These charges are collected on a copper strip glued to an insulating ceramic disk by a conductive nonvolatile silver epoxy. The strip is electrically connected to an attenuator and preamplifier which generate the resultant stop signal. This signal is processed by a constant fraction discriminator, digitized in a time to amplitude converter, and stored in a multichannel buffer before being read by a computer. This time of flight measurement allows us to detect 0.3 to $5 \mathrm{eV}$ photo-electrons with a resolution of 0.008 to $0.02 \mathrm{eV}$. 


\section{Chapter 3}

\section{Toluene / Ag(111)}

The impetus for studying the image potential state dynamics of toluene at the $\mathrm{Ag}(111)$ interface came from several years worth of research in the group. Starting in 2001, the group published a series of papers studying the IPS dynamics of the linear acenes: benzene, naphthalene, and anthracene. At some point after these were studied, it became apparent that the anthracene experiment had not actually studied anthracene, but rather a thermal decomposition product of anthracene. After a great deal of testing, it was determined that the actual system studied was a mixed system of meta, ortho, and para xylenes. In attempting to study these, an anomalously long lifetime for the IPS at 3 ML of p-xylene / $\operatorname{Ag}(111)$ was discovered ${ }^{28}$. Thus began a systematic study of the xylenes.

Previous studies of organic molecules showed that for systems in which an alkyl hydrocarbon pointed off of the surface (linear alkanes and cyclohexane), the IPS would dynamically localize. Ge et al. established that this was caused by the electron deforming its surrounds and creating a small polaron. Similar results could be expected for the xylenes. Although the exact adsorption geometry past the monolayer remains unknown, the most likely adsorption geometry is for the aromatic core to lie parallel to the silver surface at low densities, and then to tilt off pointing the ethyl groups into the vacuum. This would be consistent with adsorption of other hydrocarbons on the $\operatorname{Ag}(111)$ surface. Thus, one would intuit that the xylenes would show dynamic small polaron formation.

This, however, does not occur. In all three xylenes, the lifetime of the IPS is extended into the hundreds of femtoseconds or picosecond regime, indicating that the electron is residing on the vacuum side of the interface. This would only happen if the alkyl groups were presenting a repulsive barrier that the electron needed to tunnel through in order to return to the bulk silver. Despite the barrier and interaction with the saturated hydrocarbons, the $\mathrm{n}=1$ IPS electrons remain delocalized.

These long lived IPS electrons exhibit several interesting behaviors in p-xylene. The maximum in the TPPE signal at the bottom of the band occurs 700 fs after the pump pulse. Furthermore, there are large discrepancies in the dynamics of spectra taken at different angles. High angle spectra show no rise time at all and decay over ten times faster than low angle spectra. Spectra taken between 8 and 16 degrees show slight rise times and clear multi-exponential decays. The mechanism behind this was determined to be electronic cooling via intraband relaxation. This leads to a strongly momentum dependent TPPE decay rate when the relaxation rate exceeds the electron tunneling rate. This also explain the large rise time as electrons pile up at the bottom of the band before tunneling back into the metal and undergoing interband relaxation. 
In order to model this relaxation, Garrett-Roe et al. developed a stochastic model for simulating the electronic relaxation in k-space based on work done by Maroncelli and Flemming solvating electronic excitations ${ }^{45,46}$. In this model, an electronic wave packet is treated as a classical particle in harmonic potential. The prepared wave packet then drifts to the bottom of the potential at a rate proportional to the curvature of the well, but it also experiences a drag due to stochastic fluctuations in its environment, or friction. In the case of an IPS electron, the curvature of the band in momentum space provides the restoring force, and the motion is highly overdamped. After all, in the limit of no environmental interactions or fluctuations, an electron would remain in a given $\mathrm{k}$-state indefinitely. This classically formulated problem can be easily solved using the well known propagator for the Smoluchowski equation.

The goal in studying the toluene/ $\operatorname{Ag}(111)$ systems was two-fold. The first reason to consider this problem was to fill a gap in the literature. Computation of surface state energies and dynamics remains a computationally prohibitive problem for adsorbates. Furthermore, because of the time consuming nature of this experiment, IPS measurements have only been performed on a handful of substrates with a small number of molecules. Measuring the trends in the behavior of surface electrons across a simple chemical series such as methylation of benzene rings could help to form predictive models for how other systems may behave.

The second reason for studying toluene was to extend the friction model to dynamically localizing electrons. Previous work studying the dynamics of small polaron formation at metal/alkane interfaces ignored the effects of intraband relaxation. The reason given was that an IPS electron outside of such a large barrier should have little interaction with electrons in the bulk of the metal, and thus have no method for intraband relaxation that was nearly as fast tunneling back to the metal or dynamic electron localization which necessarily scrambles k-states. In developing the friction model, and in work by the Wolf group studying IPS electrons at the $\mathrm{N}_{2} / \mathrm{Xe} / \mathrm{Cu}$ interface, this hypothesis was debunked ${ }^{47}$. In both cases fully delocalized electrons showed extensive intraband relaxation. By estimating the distance dependent contributions to the dielectric function of the metal surface, they showed that the observed momentum dependent dynamics could be accounted for by the IPS electron interacting with damped surface plasmons and coulombic scattering off of electron-hole pair creation inside the metal. Although efforts in this area of research proved unfruitful for the toluene system, the information obtained can still be used to plan further experiments investigating the dynamics of screened IPS electrons.

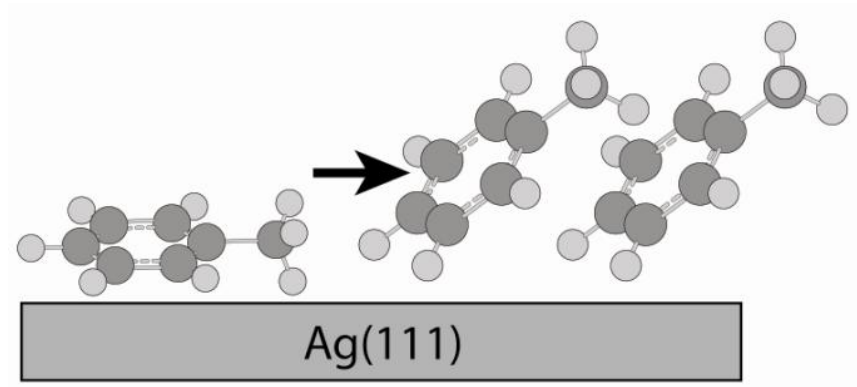

Figure 3.1 Adsorption geometry of the low density and high density monolayers of toluene to $\mathrm{Ag}(111)$. 


\subsection{Experimental}

These experiments were performed using $99.5 \%$ toluene purchased from Sigma Aldrich. Toluene was placed into a glass bulb which was attached to a side vacuum chamber. Dilute small gasses were removed by freeze-pump-thaw purification cycles. Toluene was dosed onto a $\mathrm{Ag}(111)$ crystal stored in UHV via a variable leak valve, and exposure was measured in uncorrected langmuirs. Purity of the toluene in the main chamber was confirmed by mass spec and by comparing of the main mass peaks of 92 and 93 a.m.u versus the peaks for small gases such as water, $\mathrm{N}_{2}, \mathrm{CO}$, and $\mathrm{CO}_{2}$.

\subsection{Results}

The adsorption geometry of toluene on the $\operatorname{Ag}(111)$ surface is directly known only for the monolayer. At sub monolayer coverages, the aromatic ring lies parallel to the surface as shown in figure 3.1. At a full monolayer coverage, the aromatic ring is tilted $30^{\circ}$ off the surface normal, and the methyl group points into the vacuum.

Temperature programmed desorption (TPD) was used in order to determine the proper dosing conditions for the monolayer / multilayer transition. The TPD spectrum for toluene / $\mathrm{Ag}(111)$ showed three peaks ( figure 3.2). The higher temperature broad peak between $250 \mathrm{~K}$ and $320 \mathrm{~K}$ corresponds to the monolayer. The large polarizeability of a metal surface leads to a stronger bond between it and small physisorbed molecules than between the molecules themselves. This peak is also broader than the lower temperature peak because of the phase transition between the high and low density monolayers. The lower peak at $180 \mathrm{~K}$ corresponds to multilayer desorption. The area under the monolayer peak was used to calibrate the dosing. Dosing of $3 \mathrm{~L}$ of toluene at $190 \mathrm{~K}$ was sufficient to saturate the monolayer peak, and more toluene dosed at this temperature did not stick. This was confirmed by two photon photoemission. The multilayers of toluene were dosed below 160K. The difference of $20 \mathrm{~K}$ below the onset of desorption was sufficient to ensure that no slow desorption was occurring. This was again confirmed by TPPE.

Temperature programmed desorption can yield more detailed information about the bonding energy of the molecule to the surface. Typically this is done via the Redding model. This model assumes an Arrhenius style behavior for the desorption kinetics. In this model, the rate of desorption is given by the following equation

$$
\frac{\partial \theta}{\partial t}=v \theta^{n} e^{\frac{-E_{\text {Desorption }}}{R T}}
$$

where $\theta$ is the instantaneous coverage, $t$ is the time, $\mathrm{T}$ is the temperature, $\mathrm{R}$ is universal gas constant, and $\mathrm{n}$ is the order of the desorption event. A simple substitution is used to convert the time derivative to a temperature derivative by measuring the heating rate of the sample. Most TPD spectra of small molecules that are not strongly interacting or dissociating on the surface follow first order kinetics. First order desorption kinetics give rise to a TPD peak that is asymmetrically biased to higher temperatures, and the desorption peak maximum depends on both the temperature ramp rate, $\beta$, and the maximum coverage.

The easiest way to determine the binding energy of molecule to the surface is to vary $\beta$ while keeping the initial coverage the same. This was done for the first monolayer 
of toluene on $\mathrm{Ag}(111)$. By measuring the desorption peak maximum at differing ramp rates, the enthalpy of adsorption was found to be $30 \mathrm{~kJ} / \mathrm{mol}$ according to equation (3.2):
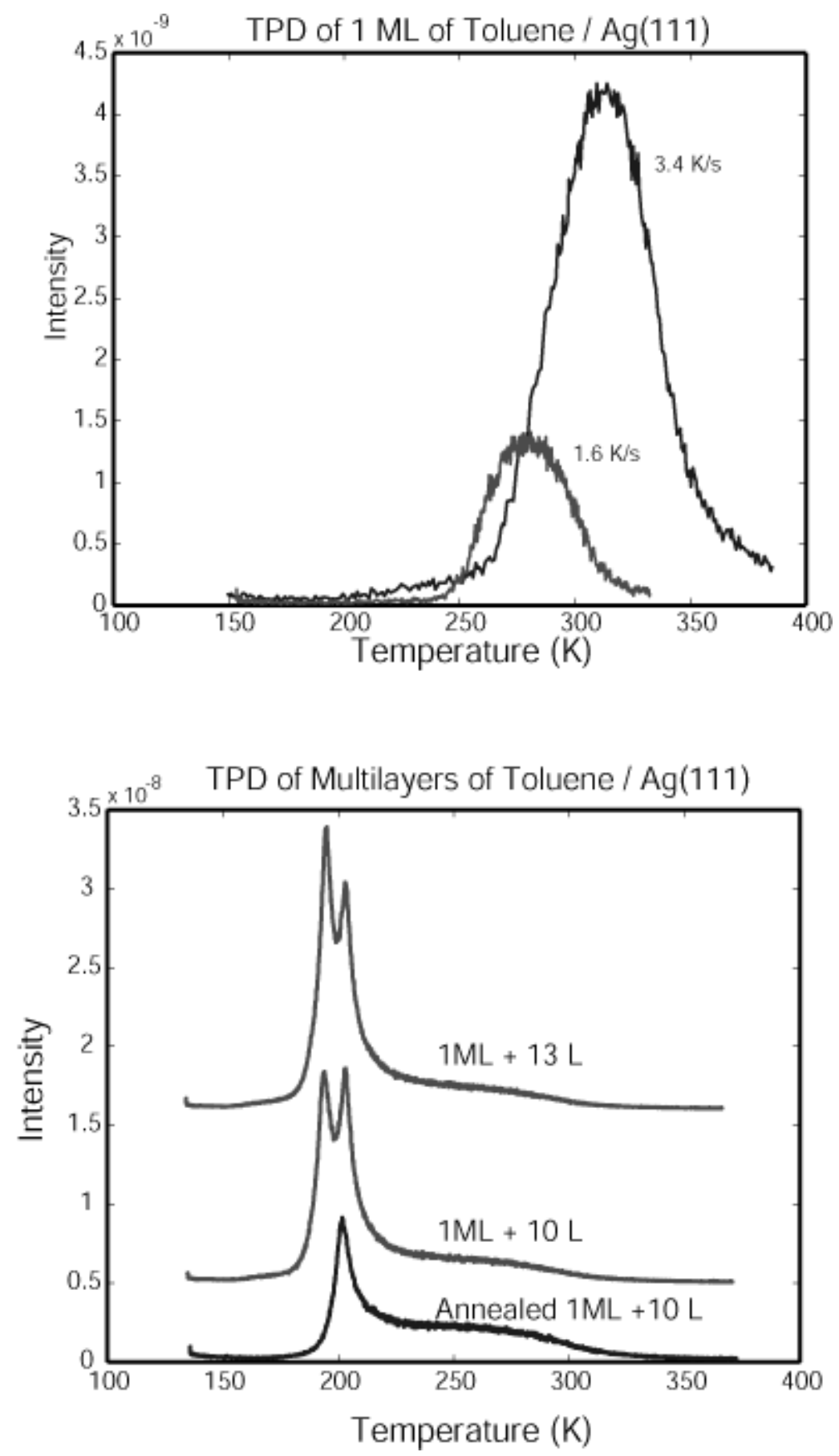

Figure 3.2 Temperature Programmed Desorption of toluene on $\mathrm{Ag}(111)$. Top panel demonstrates how the TPD spectrum changes based on heating ramp rate $(\beta)$ for the monolayer. Bottom panel shows the TPD for the multilayers. 


$$
-\log (\beta)=\alpha+\frac{\Delta H_{\text {adsorption }} * \frac{1}{2.2 * R}}{T_{\text {peak }}}
$$

where $\mathrm{T}_{\text {peak }}$ is the peak in the desorption rate, and $\Delta \mathrm{H}_{\text {adsorption }}$ is the enthalpy of adsorption. This value is consistent with other groups who have measured the binding energy at 40 $\mathrm{kJ} / \mathrm{mol}$ on more reactive surfaces.

The adsorption of the multilayer showed more complex kinetics. In order to form the most crystalline layers, multilayers of toluene were formed by dosing 3-4 L of toluene while the crystal was held at $190 \mathrm{~K}$, and then annealed at this temperature for $10-20 \mathrm{~min}$. The sample was then cooled below $160 \mathrm{~K}$ and multilayers were dosed. As shown in figure 3.2, the annealing step had a strong effect on the desorption spectroscopy. When the sample was annealed, there were only two features, the monolayer peak and a second, sharper multilayer peak at $210 \mathrm{~K}$. Without the annealing step, a third feature was introduced at even lower temperatures which was roughly equal in intensity to the multilayer peak at coverages up to1 ML $+11-12 \mathrm{~L}$. This peak represents poorly bound multilayer molecules presumably as a result of packing defects. Because the base temperature of the experimental apparatus at that time was $\sim 135 \mathrm{~K}$, this lowest energy peak experienced a slow desorption, and was unstable of the course of an experiment.

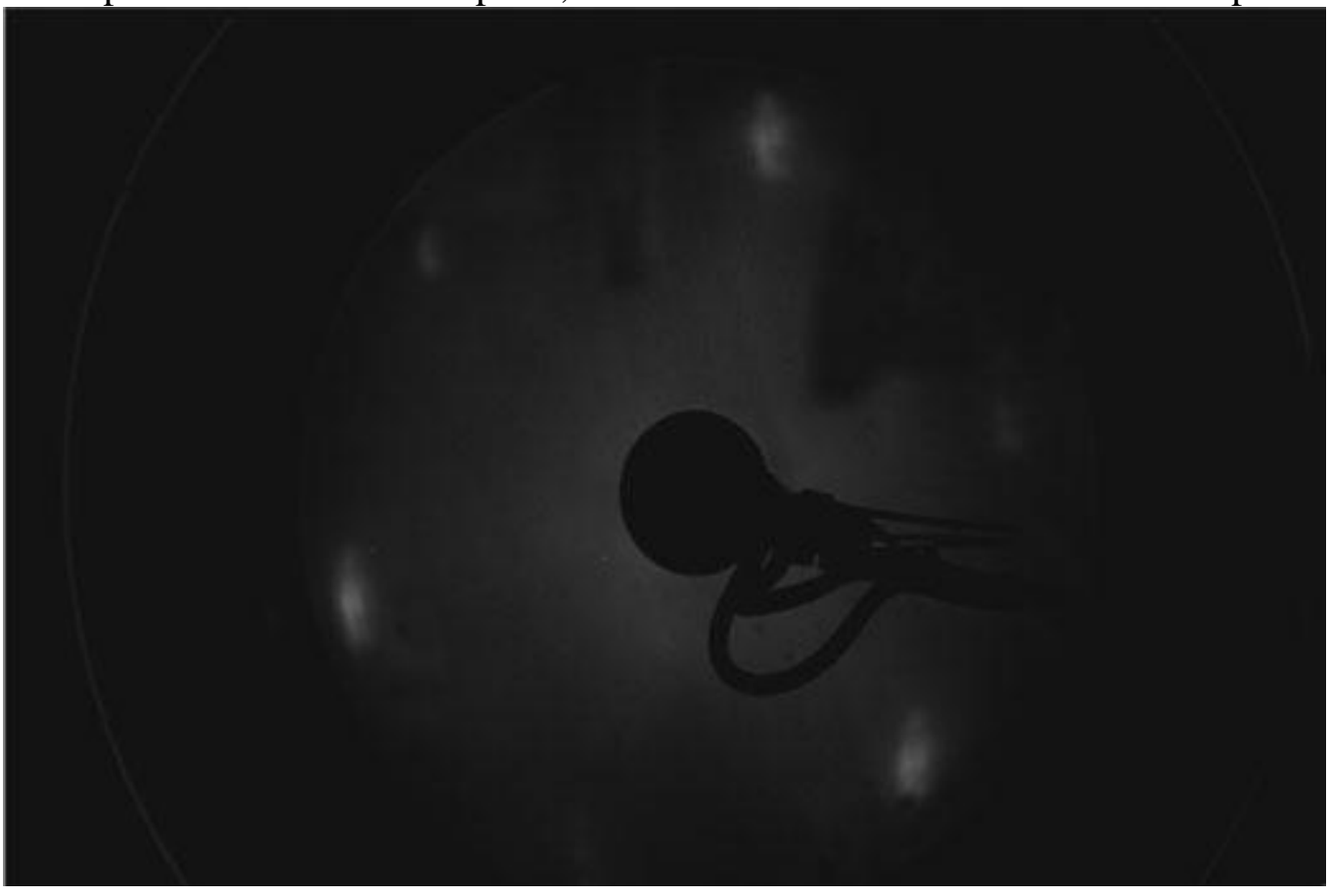

Figure 3.3 Low Energy Electron Diffraction of 1 monolayer toluene on $\mathrm{Ag}(111)$ taken at $71 \mathrm{eV}$. There are no spots visible for an ordered lattice of the toluene. The spots shown are diffraction from the underlying silver lattice. These spots are so large and misshapen because of the disordered nature of the toluene/silver interface.

Several attempts were made to measure and improve the crystallinity of the toluene / $\operatorname{Ag}(111)$ interface. These include annealing the monolayer and multilayers, and 
dosing at higher fluxes and at higher temperatures in the tail region of the desorption spectrum. Nevertheless, the interface remained a highly disorder system. Shown in figure 3.3 is a LEED image of toluene / $\mathrm{Ag}(111)$ taken at $71 \mathrm{eV}$. At lower energies, no spots were visible to indicate the multilayer adsorption geometry or unit cell. At this energy, only the silver spots are visible and they are significantly enlarged ( four times larger than clean silver) and fuzzy, indicating a disordered layer.

Shown in figure 3.4 is the TPPE spectrum of toluene dosed onto $\operatorname{Ag}(111)$. The peak at $1.36 \mathrm{eV}$ of the clean silver surface corresponds to the direct photoemission from the Shockley surface state. As the toluene adsorbs, this peak disappears as electron density from the metal gets pushed back eliminating the surface state. Once enough molecules have adsorbed to create a sufficient work function shift, the $n=1$ and $n=2$ image potential states become visible.

Fitting the spacing between the $n=1$ and $n=2$ peaks to a quantum defect parameter in equation 2.1 was used to determine the work function shift. The low quantum defect parameter for that fit $(\mathrm{a}=0.02)$ is indicative of a hydrogenic progression of states. The work function shift due to adsorption of toluene onto $\mathrm{Ag}(111)$ was determined to be $0.60 \mathrm{eV}$ yielding $\phi=3.96 \mathrm{eV}$, a value consistent with other small organic molecules such as benzene and xylenes. Because work function determination is the limiting factor in determining correct binding energies, this shift was checked against the onset of single photon photoemission. The onset of 1PPE was determined to occur at a fundamental wavelength of $633 \mathrm{~nm}$ which results in a UV photon of 3.91. The FWHM of the pulses used in the experiment were $0.04 \mathrm{eV}$, corresponding to photoemission from the high energy tail of our UV pulse.

The binding energy of the IPS was measured at $-0.81 \pm 0.03 \mathrm{eV}$ and $-0.23 \pm 0.03$ $\mathrm{eV}$ for $\mathrm{n}=1$ and $\mathrm{n}=2$ respectively. Given the lack of long range order, it is unsurprising that the TPPE spectra showed a high degree of inhomogeneity and background scattering. The FWHM of the $n=1$ is $210 \mathrm{meV}, 4.2$ times larger than for clean silver. Benzene and xylene show similar heterogeneously broadened IPS peaks for the monolayer. Despite the large degree of disorder, the electrons are delocalized with an effective mass of 1.27 \pm 0.08 and do not seem to experience any trap sites.

Dosing a monolayer equivalent of $3 \mathrm{~L}$ toluene below $160 \mathrm{~K}$ results in the low energy shoulder of the $n=1$ peak shifting to lower energies as shown in figure $3.5 \mathrm{~A}$. This feature is marked with an asterisk. Both peaks remain present in the spectrum past 4 monolayer equivalents. These spectra were taken at $650 \mathrm{~nm}$, which was near the lower limit of obtainable spectra without appreciable UV photoemission. The dynamics of both features were measured for all four coverages. The asterisked peak showed the same dynamics of the monolayer regardless of actual coverage. The dynamics of the main feature showed increasing lifetime as a function of coverage.

\subsection{Discussion}

Interpretation of the TPPE spectra for the toluene monolayer is quite simple. The assignment of both peaks to image potential states is unambiguous. The electronic structure of toluene is very similar to benzene, with strong optical absorption not occurring until $4.35 \mathrm{eV}$ in 
the liquid phase. Given that the highest energy photons used in these experiments were $3.91 \mathrm{eV}$, we can safely discard any contribution from direct molecular excitation. A wavelength survey was done to confirm that all spectral features shown in the spectra are pumped by the UV pulse and photo-emitted by the visible. The 50 fs lifetime for the $n=1$ IPS is also consistent with an electron residing near the $\mathrm{Ag}(111)$ surface.
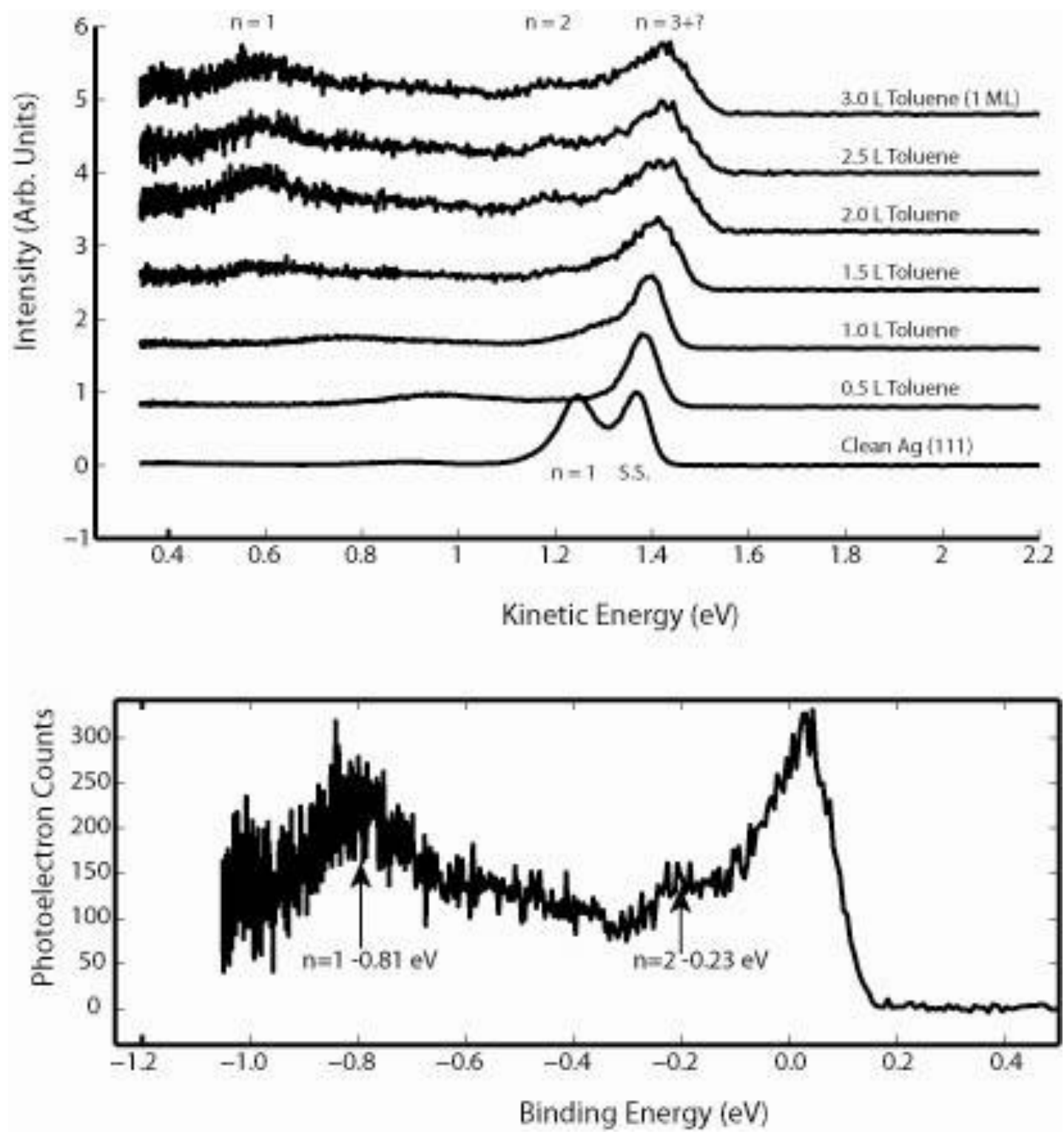

Figure 3.4 Two photon photoemission spectra of toluene/ $\mathrm{Ag}(111)$. The top panel shows the spectral evolution of the surface upon exposure to toluene. The bottom panel shows the spectrum of 1 monolayer of toluene/ $\mathrm{Ag}(111)$ with the measured binding energies for the image potential states.

Interpreting the results of the multilayer spectra are significantly more complicated due to the presence of multiple peaks in the small region. Figure 3.5A shows the TPPE spectrum of increasing coverages of toluene all taken at the same 
detector bias. The asterisked peak moves to lower energy, and does not form a hydrogenic progression with the other peak, making it difficult to interpret the two features as the $n=1$ and $n=2$ IPS. Furthermore, the only known mechanism for $n=1$ IPS binding at over $0.97 \mathrm{eV}$ below the vacuum level is for the IPS to interact with a highly positive electron affinity level. It is expected that the binding energy of the $n=1$ for toluene should be near the binding energy of benzene and xylene since they are electronically and structurally similar. The $n=1$ IPS has a measured binding energy of $0.68 \mathrm{eV}$ for multilayers of benzene and $-0.69 \mathrm{eV}$ to $-0.44 \mathrm{eV}$ for multilayers of xylenes.

The key to interpreting these spectra comes from the differing dynamics for the two features. The dynamics in figure 3.5C were fit by first fitting the intensities of the TPPE spectra at each time point to one Voigt function for the monolayer and two for the multilayer. These intensities were then fit to by convoluting a Gaussian of $120 \mathrm{fs}$ representing the pulse cross correlation with an exponential rise and decay. The pumpprobe overlap time was not allowed to vary by more than \pm 20 fs. The low energy featured has an exponential decay time of 50 fs regardless of exposure.

The interpretation of long lifetimes in screened image potential states is often understood as the molecules presenting a repulsive barrier through which the electron must tunnel in order to return to the metal surface. In dielectric continuum theory this occurs whenever the electron affinity level of the material is greater than zero (i.e. repulsive). Here, this interpretation is used to infer that the asterisked feature is in fact the $n=1$ IPS for patches of monolayer. The reasoning is that it maintains the dynamics of the monolayer, while the other feature shows a strongly increasing lifetime as a function of exposure.

The decrease in energy from the monolayer binding energy of $-0.81 \mathrm{eV}$ is interpreted as a shift in work function rather than a shift in binding energy. When the work function of the sample changes, a corresponding voltage bias needs to be applied to the detector to ensure that the electron remains in a field free region during its flight time. Indeed, the wavelength of the fundamental needed to be increased to $650 \mathrm{~nm}$ to avoid one photon photoemission which corresponds to a work function shift of at least $0.1 \mathrm{eV}$.

Since the layers show no signs of long range order and are being dosed at cryogenic temperatures, it is not surprising that a disordered material with patches of monolayer exposed could form. Figure 3.5B shows the same series of coverages as 3.5A, but here the energy axis of each spectrum has been adjusted to place the $n=1$ IPS for the monolayer at $-0.81 \mathrm{eV}$. From this, the binding energies of the $n=1$ for multilayer exposures are estimated to be $-.57,-.63$, and - .622 $\pm 0.05 \mathrm{eV}$ for 1,2 , and 3 monolayer equivalent exposures respectively. It is important to remember that these are units of exposure, rather than actual coverages, and the thickness of each patch of multilayer remains undetermined in the experiment.

Having measured the energetic and dynamics of the IPS at the bottom of the band $\left(\mathrm{k}_{/ /}=0\right)$, an attempt was made to analyze the kinetics of localization. In order to study momentum dependent relaxation pathways, the electron needs to have a lifetime much greater than the pump probe cross correlation. For this reason, the monolayer $+9 \mathrm{~L}$ coverage was chosen for exploring the relaxation pathways of the IPS electron. Thicker 
A)

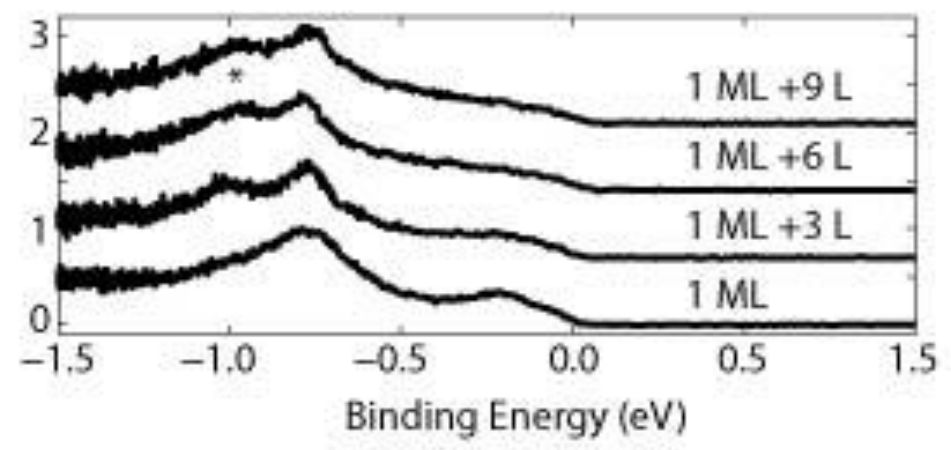

B)

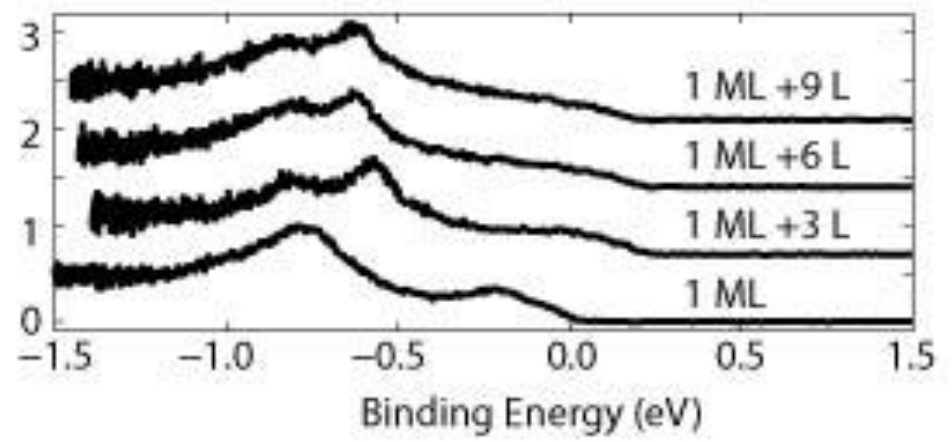

C)

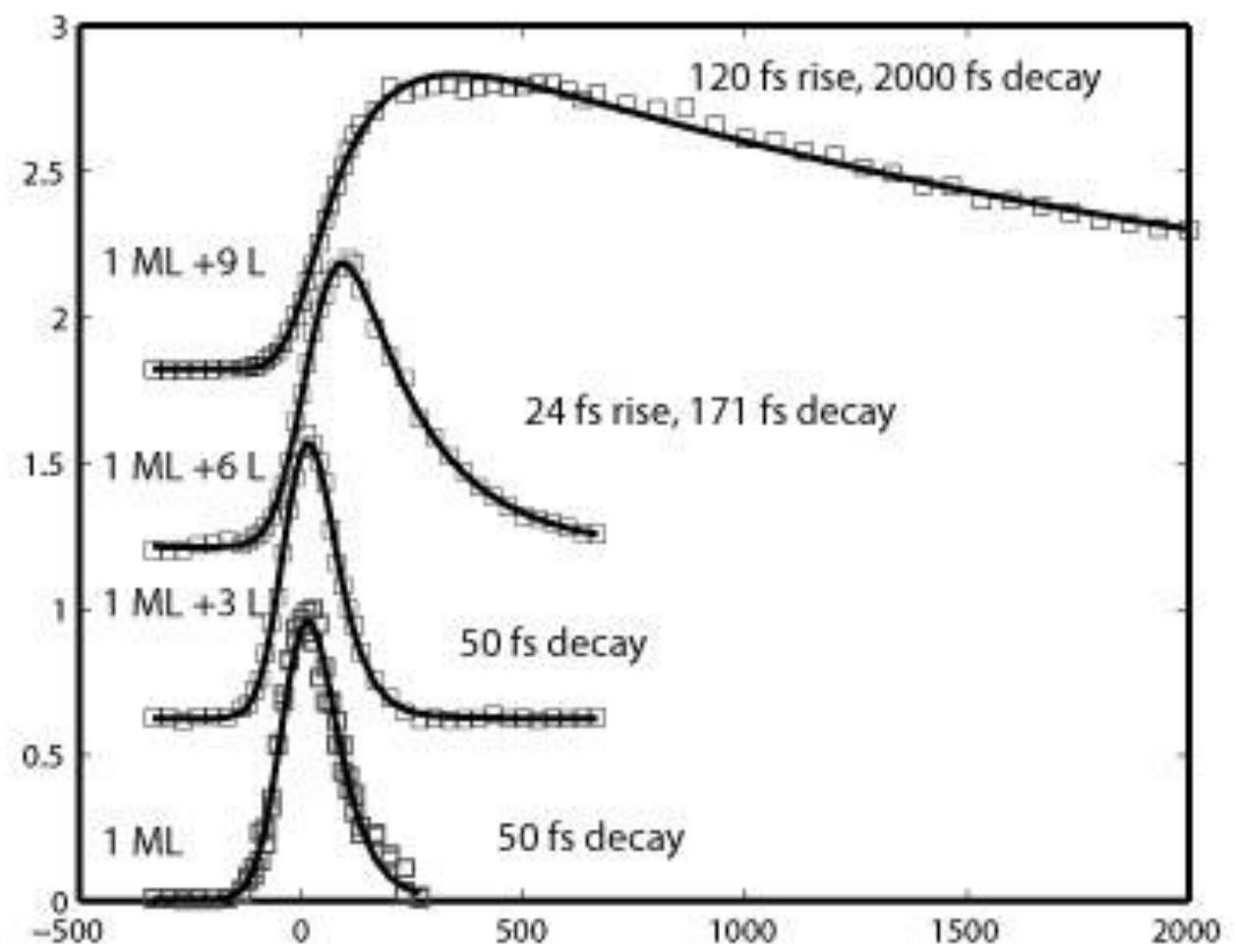

Figure 3.5 A) Two photon photoemission spectra of increasing coverages of toluene dosed at $160 \mathrm{~K}$. The energy axis is uncorrected for work function shifts. B) Same spectra as in panel A, but with the energy of axis for each spectra modified to center the asterisked peak at the monolayer $n=1$ binding energy of $-0.81 \mathrm{eV}$. C) Dynamics of the $\mathrm{n}=1$ IPS electron at differing coverages. 
coverages were not investigated because although the lifetimes continued to increase, the signal to noise decreased rapidly.

Angle resolved dynamics were obtained by varying the angle of sample with respect to the detector in $2^{\circ}$ increments. At each angle, four to eight spectra were taken with the stage moving forwards and backwards in order to make sure that the observed dynamics were not a result of laser drift. For population analysis between different angles, the laser power was kept constant between angles, and the crystal was periodically returned to the zero degree position to make sure that the photo-electron counts remained constant. When analyzing the data, the intensity of each spectrum was scaled by $1 / \cos ^{4} \theta$ to account for the screening of light parallel to the surface by the metal's electrons.

The spectra at each angle showed an initially delocalized peak image potential state for the multilayer. Over the first several hundred femtoseconds and at high angles, a new lower energy peak appeared at $-0.62 \mathrm{eV}$, the same position as the delocalized $n=1$ at zero degrees. By

$500 \mathrm{fs}$, there is only one peak visible at each angle, which corresponds to the fully localized electron with an effective mass of $\mathrm{m}^{*}>50 \mathrm{~m}_{\mathrm{e}}$. A sample of these dynamics is shown in figure 3.6. The lifetimes of the delocalized features varied by nearly an order of magnitude, making the toluene/Ag(111) interface a decent candidate for attempting to separate intraband relaxation from localization.

The model used to analyze the intraband relaxation component was developed by Sean Garrett-Roe has already been described elsewhere at length. It is summarized here only to provide context for my own modifications.

IPS electrons are treated as bound particles in the direction normal to the surface, and as nearly free electrons parallel to the surface, with definite momenta parallel to the surface given by $\hbar \mathbf{k}_{/ /}$. The band structure of the system is nearly free electron like with a parabolic dispersion relation given by :

$$
E\left(\mathbf{k}_{/ /}\right)=\frac{\hbar^{2} k^{2}}{2 m^{*}}
$$

where $\mathrm{m}^{*}$ is the standard effective mass of an electron in the band. In effect, this defines a parabolic potential energy surface in k-space. In the absence of any other forces, an electron would stay in its given k-state indefinitely. The IPS electron, however, is coupled to a bath of electronic and phonon modes of the adsorbates/metal system. The effect of this coupling is to slow the velocity of the electron parallel to the surface by dissipating energy to the bath. This deceleration of the electron in real space is equivalent to dragging the electron to the bottom of the band in k-space. Rather than attempting to calculate the individual mechanism of IPS/bath coupling, we will use the simplest model, stochastic friction. 

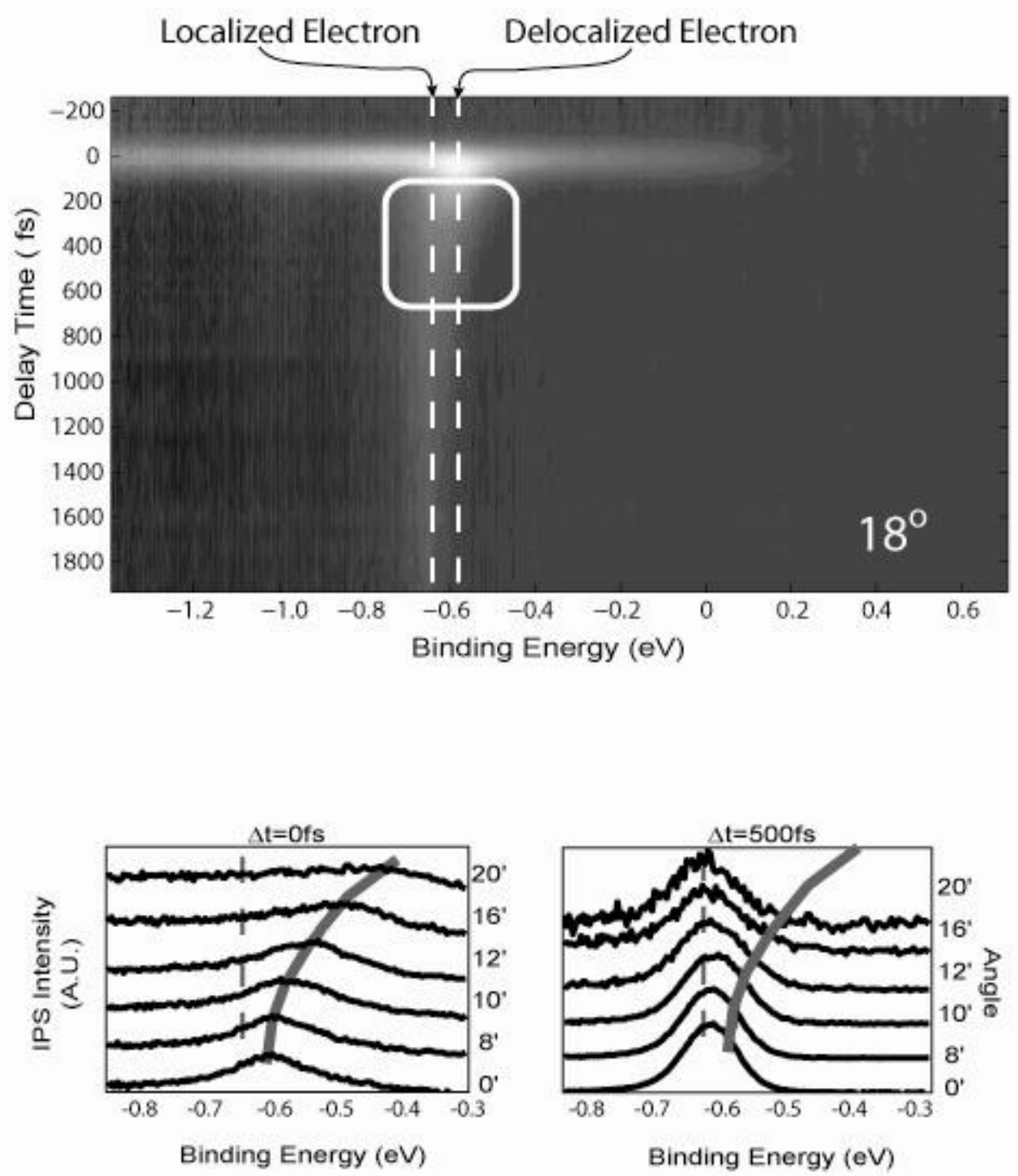

Figure 3.6 Angle resolved dynamics of the $n=1$ IPS at multilayer patches of toluene/ $\mathrm{Ag}(111)$. The top panel is a series of dynamic scans taken over 64 time points with the sample held at $18^{\circ}$. The dotted lines are guides to the eye for the energetic positions of the localized and delocalized electrons. The white box shows the temporal region where both a localized and delocalized state are visible. 
Coupling to the bath results in a random walk of the electron and gives rise to both a linear frictional force:

$$
F_{\text {friction }}=-\Gamma_{x} * \dot{x}
$$

where $\Gamma_{\mathrm{x}}$ is the coefficient of friction in real space and $\dot{x}$ is the electron's velocity, as well as diffusive motion related to friction and the temperature of the ensemble:

$$
D_{x}=\frac{k_{b} T}{\Gamma_{x}}
$$

where $\mathrm{D}_{\mathrm{x}}$ is the diffusion constant, $\mathrm{k}_{\mathrm{b}}$ is Boltzmann's constant, and $\mathrm{T}$ is the absolute temperature of the bath. Simple physical considerations and unit analysis show that the friction in real space is inversely proportional to the friction in $\mathrm{k}$-space:

$$
\Gamma_{k}=\frac{\hbar^{2}}{\Gamma_{x}}
$$

In this model then, the band structure of the IPS is like a harmonic potential energy, and the friction is like an overdamped restoring force. For a given initial distribution of overdamped oscillators, the population dynamics $\mathrm{p}\left(\mathrm{k}_{\mathrm{x}}, \mathrm{t}\right)$ has an analytic solution provided that the friction is Markovian (i.e. has no memory) ${ }^{48}$. Using the appropriate Green function for a momentum distribution:

$$
G\left(k_{x}, t \mid k_{x 0}, t_{0}\right)=\sqrt{\frac{1}{2 \pi \sigma_{k x}^{2}(t)}} e^{\frac{-\left(k_{x}-<k_{x}>(t)\right)^{2}}{2 \sigma_{k x}^{2}(t)}}
$$

The population as a function of time and initial distribution is given by:

$$
p\left(k_{x}, t \mid k_{0}, t_{0}\right)=\int_{-\infty}^{\infty} G\left(k_{x}, t \mid k_{x 0}, t_{0}\right) p\left(k_{0}, t_{0}\right) d k_{x 0}
$$

Where $\sigma_{\mathrm{kx}}$ is the time dependent width of the temperature dependent propagator and $\left\langle\mathrm{k}_{\mathrm{x}}\right\rangle(\mathrm{t})$ is the time dependent average of the distribution. These can be written as:

$$
\begin{gathered}
\sigma_{k x}^{2}(t)=\frac{D_{k x}\left(1-e^{-2 B_{k x}\left(t-t_{0}\right)}\right)}{B_{k x}} \\
<k_{x}>(t)=k_{x 0} e^{-B k_{x}\left(t-t_{0}\right)}
\end{gathered}
$$

Here, the $\mathrm{D}_{\mathrm{kx}}$ is the diffusion constant in k-space of the electron, and is given by:

$$
D_{k x}=\frac{k_{b} T}{\Gamma_{k x}}
$$

and $\mathrm{B}_{\mathrm{kx}}$ is simply a measure of the force dragging an electron to the bottom of the band and depends on the curvature of the band and the frictional constant as given below.

$$
B_{k x}=\frac{\hbar^{2}}{m^{*} \Gamma_{k x}}
$$

At this point, the physical picture of the solution can be adequately described. At time $t=0$, a population of excited electronic states is created. This population can be experimentally determined via angle-resolved two photon photoemission at pump-probe, temporal overlap. As time progresses, the high momentum IPS electrons exchange energy with the bath (electronic and phonon modes of the adsorbates and/or metal), 
dissipating energy and drifting to the bottom of the band. Due to the random nature of thermal fluctuations, however, the probability distribution does not collapse to $\mathrm{p}\left(\mathrm{k}_{\mathrm{x}}, \mathrm{t}=\infty\right)$ $=\delta(0)$, but rather to a Boltzmann distribution of electron velocities. Extending this one dimensional model to a 2D model in cylindrical coordinates as appropriate to electrons at a surface is an algebraic matter and the details are shown in the original publications ${ }^{27,48}$.

By construction, this model for intraband relaxation obeys the fluctuation dissipation theorem; however, as stated it does not allow for actual population decay back into the metal. Electron tunneling dynamics can be simply accounted for by a first order correction to the propagator, leading to exponential decay of the population in each state $^{49}$. The resultant, and final equation governing the kinetics is then:

$$
p\left(k_{x}, k_{y}, t \mid k_{x 0}, k_{y 0}, t_{0}\right)=\iint_{-\infty}^{\infty} d k_{x 0} d k_{y 0}\left[G\left(k_{x}, k_{y}, t \mid k_{x 0}, k_{y 0}, t_{0}\right)-\kappa_{0}\left(k_{x}, k_{y}\right)\right] p\left(k_{x 0}, k_{y 0}, t_{0}\right)
$$

This equation can then be numerically propagated if the initial population distribution is known from two photon photoemission measurements. In this formulism, the decay rate back to the metal is approximated as constant. Detailed work has shown that the tunneling rate is affected by the energetic proximity to the conduction band edge $^{50,51}$. At higher momenta, there is an increased probability of an electron scattering into the conduction band. Unfortunately, calculating this dependence for molecular adsorbates containing several atoms is computationally unfeasible at an atomistic level, and gives incorrect values at the dielectric continuum model level. So here we have neglected the dependence of the tunneling rate upon parallel momentum. The fit of the dynamics of the delocalized toluene data to this model is shown in figure 3.7 with a friction constant of $\Gamma=4 \times 10^{-14} \mathrm{~kg} / \mathrm{s}^{2}$.
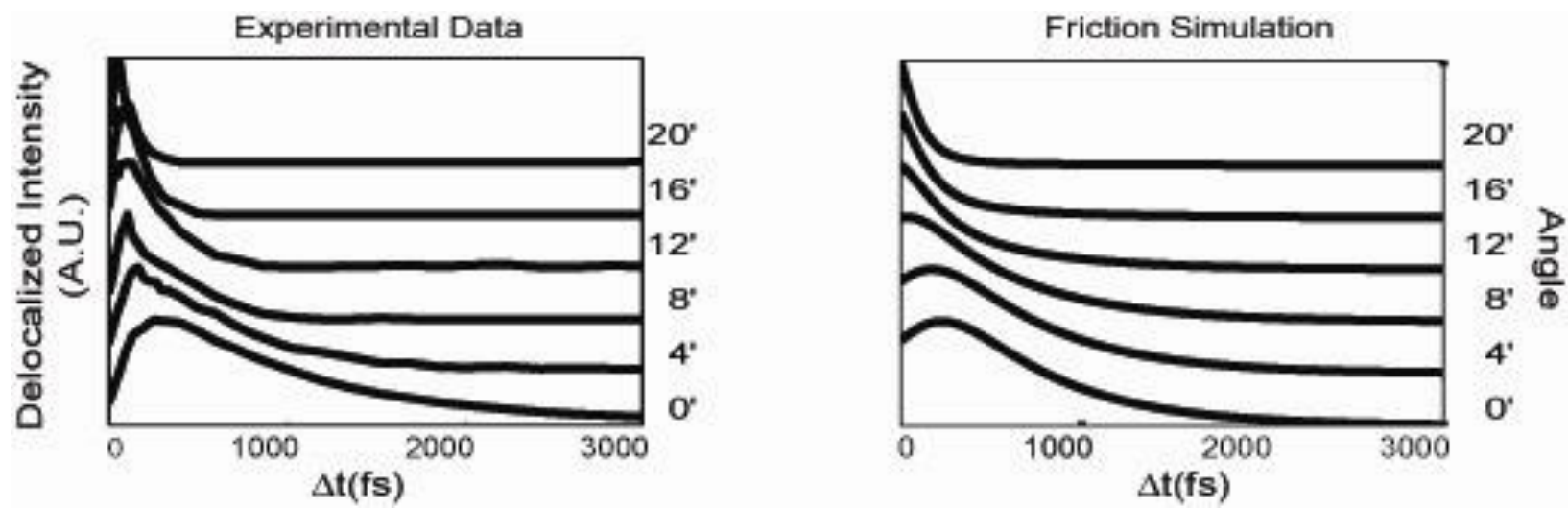

Figure 3.7 Left panel shows the experimentally measured dynamics of the delocalized $n=1$ IPS at multilayer patches at different angles. Right panel shows the best fit to that data using the friction simulation discussed here with a friction constant of $\Gamma=4 \times 10^{-14} \mathrm{~kg} / \mathrm{s}^{2}$.

This model accurately captures the behavior of the delocalized peak with a friction constant that is slightly larger than that for the p-xylene at $\Gamma_{\mathrm{p} \text {-xylene }}=2 \times 10^{-14} \mathrm{~kg} / \mathrm{s}^{2}$. The magnitude of the friction is based on the distance to the metal and the ability of the electron to scatter off of electron hole pairs and damped plasmons. Larger friction 
implies that that the long lived toluene IPS is farther from the metal surface. This is consistent with the hypothesis that there are large thick islands of toluene present on the surface.

The next step in analyzing the angle resolved dynamics was to account for electron population transfer from the delocalized state into the localized state. Luckily, the way to do this within the model was already established by Maroncelli et al. and is shown in equation 3.13:

$$
p\left(k_{x}, k_{y}, t \mid k_{x 0}, k_{y 0}, t_{0}\right)=\iint_{-\infty}^{\infty} d k_{x 0} d k_{y 0}\left[G\left(k_{x}, k_{y}, t \mid k_{x 0}, k_{y 0}, t_{0}\right)-\kappa_{0}\left(k_{x}, k_{y}\right)-\Delta S\left(k_{x}, k_{y}\right] p\left(k_{x 0}, k_{y 0}, t_{0}\right)\right.
$$

In this equation, the term $\Delta \mathrm{S}$ acts as a population transfer function. Judicious selection of the form of this function should allow for testing of rate of localization while explicitly accounting for the background intraband component to the momentum dependent population relaxation. Since small polaron formation has already been shown to be isomorphic with Marcus electron transfer theory, the natural selection for a sink function is the standard Marcus theory transfer rate:

$$
\Delta S\left(k_{/ /}\right) \propto \frac{1}{\sqrt{4 \pi \lambda k_{B} T}} \exp \left(-\frac{(\lambda+\Delta G)^{2}}{4 \lambda k_{B} T}\right)
$$

where $\Delta \mathrm{G}$ is the energy difference between the bottom of the delocalized and energy of the localized peak, and $\lambda$ then equals the delocalized band structure $\left[\mathrm{E}\left(\mathrm{k}_{/ /}\right)-\mathrm{E}(0)\right]+\Delta \mathrm{G}$ for a fully non dispersive final state.

The problem with this method lies in the inability to distinguish a localized electron from a delocalized electron near zero degrees. Although the experimental apparatus is capable of resolving individual electrons and the center of a peak to a resolution of less than $.015 \mathrm{eV}$, it is in capable of resolving multiple peaks in such a region. This is illustrated in figure 3.8 where the binding energy at zero degrees for the $\mathrm{n}=1$ IPS is measured over time, as is the FWHM of the fitted peak's width. Although the experiment is capable of measuring the $20 \mathrm{meV}$ shift from the $\mathrm{t}=0$ binding energy to the binding energy at 2 picoseconds, there is no way to resolve two peaks at differing by only that amount. At various time delays around 200 to 400 seconds and at angles greater than $12^{\circ}$, it is obvious that both the delocalized and localized peak exist at the same time, and it does not fit well to a single peak. Below $12^{\circ}$, there is no statistical confidence fitting the spectra to one wider peak or two narrow peaks artificially constrained to be separated. Since it is primarily the peaks below $12^{\circ}$ that are thermally populated following intraband relaxation, reasonable changes in the functional form of $\Delta S$ can be masked by making small changes in the friction constant $\Gamma$. Several other $\Delta \mathrm{S}$ dependencies on $\mathrm{k}_{/ /}$were tried including $\Delta \mathrm{S}$ that were independent, linearly dependent and parabolically dependent on $\mathrm{k}_{/ /}$, however none were satisfactory. In order to use this method to truly disentangle the dynamics of intraband cooling and electron localization, the localized peak must be sufficiently energetically separated from the delocalized peak that their dynamics can be unambiguously measured. 


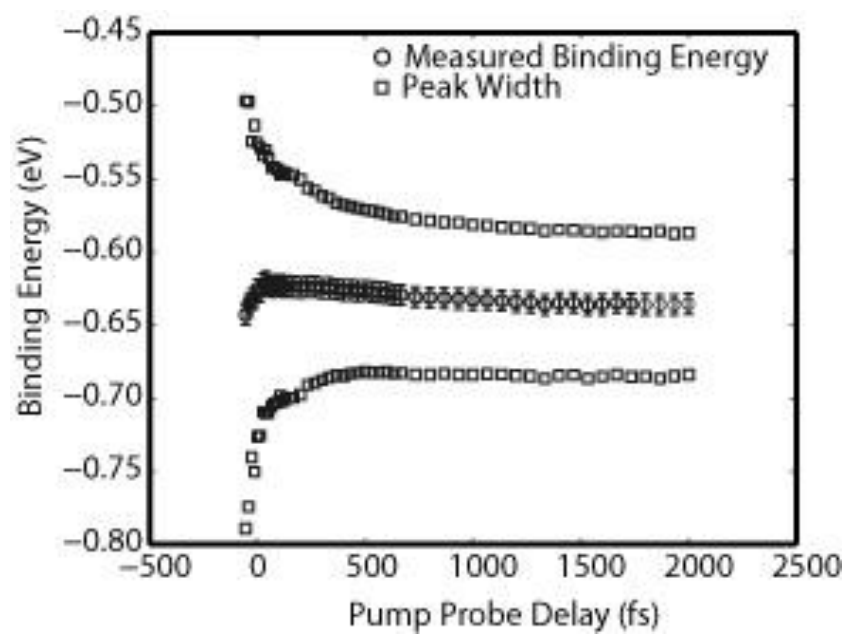

Figure 3.8 Measured peak energy of the $n=1$ IPS over multilayer patches of toluene and the peak width. The peak width was determined by the sum of the Gaussian and Lorentzian FWHM line widths in the Voigt fit.

\subsection{Conclusions}

Temperature programmed desorption and two photon photoemission have been used to study the adsorption of toluene at the $\operatorname{Ag}(111)$ interface and its interfacial electron dynamics. For the monolayer, toluene has two resolvable image potential states bound at 0.81 and $0.23 \mathrm{eV}$ below the vacuum, both of which decay with a time constant of $50 \mathrm{fs}$. These results are similar to results obtained for benzene and xylene on $\mathrm{Ag}(111)$. Multilayers of toluene showed patchy growth, leaving elements of the wetting monolayer exposed. The patches of multilayer also showed an image potential state whose binding energy varied between -0.63 and -0.57 depending on coverage. The lifetime of these multilayer IPS electrons increased rapidly with coverage, leading to a state with a two picosecond lifetime. Attempts were made to apply a modified stochastic model for electron relaxation in order to verify the mechanism for electron localization in this system, however they were not successful. 


\section{Chapter 4}

\section{Charge localization by alkyl side chains in Sexithiophene and DH6T}

\subsection{Introduction}

Charge and exciton dynamics play a dominant role in the performance and efficiency of photovoltaics, field effect transistors, and light emitting diodes. Molecular organic and polymeric materials have potential for production of low cost and easily processed devices, however, they are more susceptible to charge localization and reduced mobility as compared to their inorganic counterparts ${ }^{52}$. The low dielectric constants and lighter atoms in these materials increases the potential for lattice distortion by a moving electron, resulting in the formation of a small polaron. Conduction through hopping of small polarons can result in a mobility decrease of $10^{4}$ relative to band transport, and the energy lost to the charge stabilization reduces the open circuit voltage and efficiency of organic photovoltaics.

Despite agreement on the presence of conduction through small polarons in organic semiconductors, there is little research on their formation. The rate, magnitude, and mechanism of charge stabilization are poorly understood and expected to vary with molecular identity. Molecular and orientational disorder in organic thin films has limited study of their band structures. Recent progress has been made in growing linear aromatic semiconductors with high crystallinity, allowing spectroscopic studies of electronic orbitals using angle resolved photo-emission spectroscopy (ARPES) ${ }^{53,54}$. Studies using linear acenes as a model molecule have revealed a mixture of states exhibiting either delocalized or localized characters perpendicular to the molecular axis, while parallel to the axis the bands disperse with a periodicity of twice the molecular monomer length. The presence of these types of delocalized and dispersive states opens the possibility for utilizing non-hopping charge transport in highly oriented systems and devices.

Alkyl substitution of organic semiconductors and polymers is a common technique for improving morphology, charge mobility and efficiency of devices. This substitution is used to increase solubility in solvents for spin casting and to improve solid state miscibility with fullerenes for use in photovoltaics and is most famous in the P3HT/PCBM class of organic solar cells ${ }^{55-59}$. Previous studies have shown that homophilicity of the polymer and ligands can lead to better intermolecular overlap in spin 
cast systems. Increased ordering is attributed to competitive van der Waal's interactions between alkyl and aromatic moieties, resulting in the grouping of similar functional groups. This decreases rotational disorder while still leaving pi-stacked channels for high charge mobility, often resulting in 2D conduction channels. Unfortunately, previous studies have found that ultrathin films of alkane chains of varying lengths all rapidly localize initially delocalized charges, a behavior attributed to the methyl rocking motion at $750 \mathrm{~cm}^{-139}$. Thus, even in systems in which alkyl substitutions improve charge mobility, they may also impose an additional upper bound on device performance by creating localized, persistent, self-trapped charges.

\subsection{Experimental}

$\alpha$-Sexithiophene and $(\alpha, \omega)$-dihexyl-sexithiophene were purchased from Sigma Aldrich. These were loaded into a ceramic crucible inside of a Knudsen cell and degassed at $25 \mathrm{~K}$ below their dosing temperature for greater than 24 hours to remove small gas impurities such as $\mathrm{N}_{2}, \mathrm{CO}_{2}$, and $\mathrm{O}_{2}$ and until the pressure was below $3 \times 10^{-8}$ torr. The samples were further degassed before introduction into the main chamber at 20 $\mathrm{K}$ above their dosing temperature in order to ensure that no impurities from the heating filaments were entering the main chamber.

It was discovered during the experiment that these materials showed a rapid rate of degradation when exposed to ultrafast pulses, and that this degradation was faster for multilayers than the monolayer. This degradation shifted the energy of all peaks in the spectrum to higher energy, increased their spectral width, and decreased the intensity of the signal. In order to avoid this, the rate of degradation was measured by taking consecutive TPPE spectra with 20 second acquisition times over the course of 30 min to two hours. No detectable damage was done to the layer within one to two minutes, and so beam exposure time was limited to 30 seconds per scan (single time point and single angular point) while acquiring the dynamic band structure before the sample was translated. The exact mechanism was not determined for the degradation, but was most likely either laser induced sample desorption or multi photon absorption causing high energy chemistry to link molecules on the surface. Powers at the sample $\sim 3 \mu \mathrm{W}$ for the visible pulse and $\sim 200 \mathrm{nW}$ for the UV pulse.

\subsection{Results}

Here, we present ultrafast time and angle resolved two photon photoemission of ultrathin films of the linear organic semiconductors sexithiophene (6T) and its alkylated counterpart $\alpha-, \omega$-dihexyl-sexithiophene (DH6T) on $\operatorname{Ag}(111)$. These were selected because they model the polythiophene and alkylated polythiophene molecules used in many organic solar cells ${ }^{60}$ and because their adsorption and crystallinity have been previously explored $^{61,62}$. Layer-by-layer growth was achieved via molecular beam epitaxy from a Knudsen cell attached to the main chamber. The growth rate of the layers was determined from changes in the 2PPE spectra, as well as low energy electron diffraction patterns (LEED). LEED patterns for both 6T and DH6T matched the known adsorption geometries for mono- and mulitilayers on $\mathrm{Ag}(111)$ which are presented in 
Figure 4.1. Dosing conditions including flux and substrate temperature were altered to give the sharpest diffraction spots and least background signal in the 2PPE spectra.

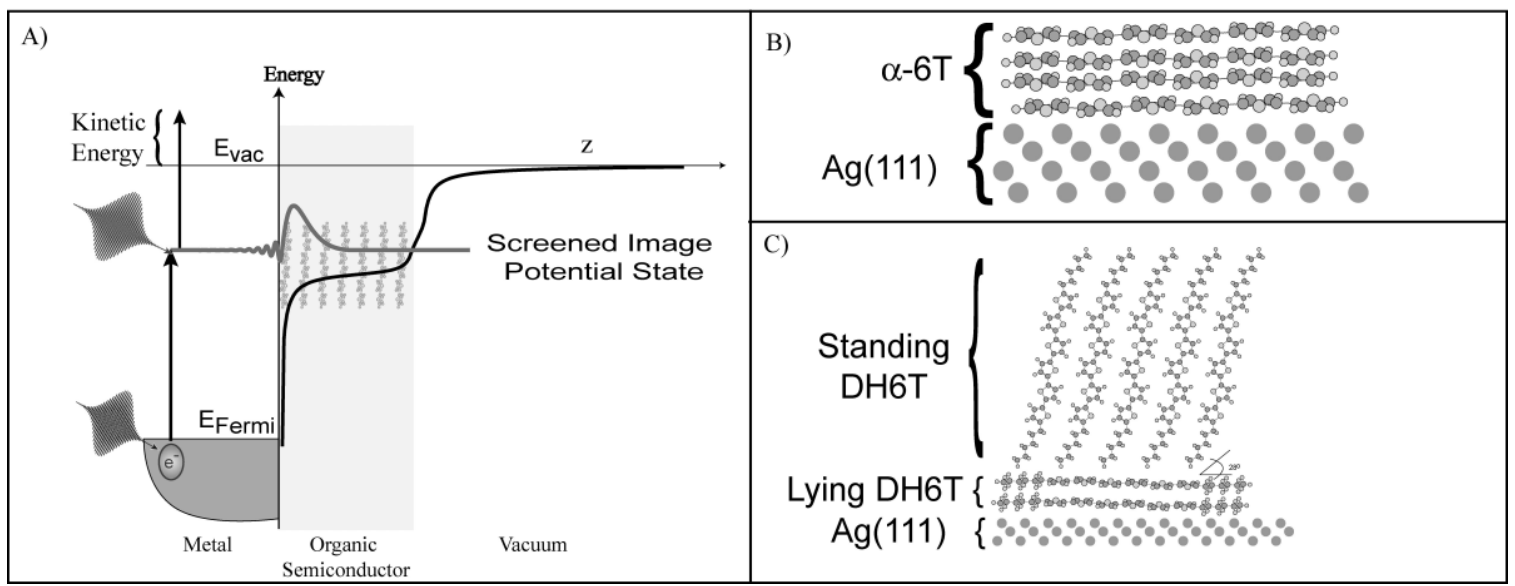

Figure 4.1: A) Schematic of the two photon photoemission process. The first UV pulse excites an electron from at or below the Fermi level of the metal into an image potential state which spatially overlaps with the adsorbate and the vacuum. A second visible pulse then photoemits the electron and the kinetic energy of the photoelectron is measured thus determining the binding energy of the intermediate state. The photon energies of both pulses are kept below the work function of the material to eliminate contributions due to one photon photoemission. B) \& C) The known adsorbtion geometries for sexithiophene and dihexyl-sexithiophene on the $\mathrm{Ag}(111)$ surface.

Two peaks are present in the 2PPE spectrum of 6T / $\operatorname{Ag}(111)$ at all coverages (Figure 4.2A). These peaks are the first and second image potential states (IPS) at the semiconductor/metal interface. Image potential states are a hydrogenic series of electronic states derived from the high polarizability of metallic systems bound in the surface normal direction. The average distance from the metal surface for the first IPS is only 3 angstroms, making it very sensitive to changes in its local electronic environment due to adsorbates. Parallel to the surface, these states are delocalized and behave like nearly free electrons for bare surfaces. In the presence of an adsorbate, the lateral extent of an the IPS can either be highly localized or delocalized, depending on its interaction with the layer. They shift continuously from the energy position of the clean Ag peaks to lower energy as the workfunction decreases. Adsorption of the first monolayer of 6T lowers the workfunction by $0.68 \mathrm{eV}$ to $3.89 \mathrm{eV}+/-0.05 \mathrm{eV}$. These states are located at $3.06 \mathrm{eV}$ and $3.64 \mathrm{eV}$ above the Fermi level for the first monolayer, or a binding energy of $-0.83 \mathrm{eV}$ and $-0.25 \mathrm{eV}$. Energetic ordering of the states was determined by changing the wavelength of the probe pulse and measuring the change in photoelectron energy. The increased binding of the $n=1$ image potential state at the 6T/Ag(111) interface compared to the clean silver binding energy of $-.77 \mathrm{eV}$ is consistent with other organic semiconductor metal interfaces that have a high electron affinity $(>2 \mathrm{eV})^{30,63,64}$.

Increased coverage had a negligible affect on the workfunction or binding energy of the $n=1$ peak. The second IPS peak broadens and decreases in intensity with increasing thickness, eventually becoming indiscernible by 4 - 5 monolayers. The measured lifetimes of both states remained below $30 \mathrm{fs}$ for all coverages (Figure 4.2B). 
This implies that the wave function of screened IPS maintains similar overlap with the metal substrate as bare IPS whose lifetime is 22 fs for $\operatorname{Ag}(111)$.
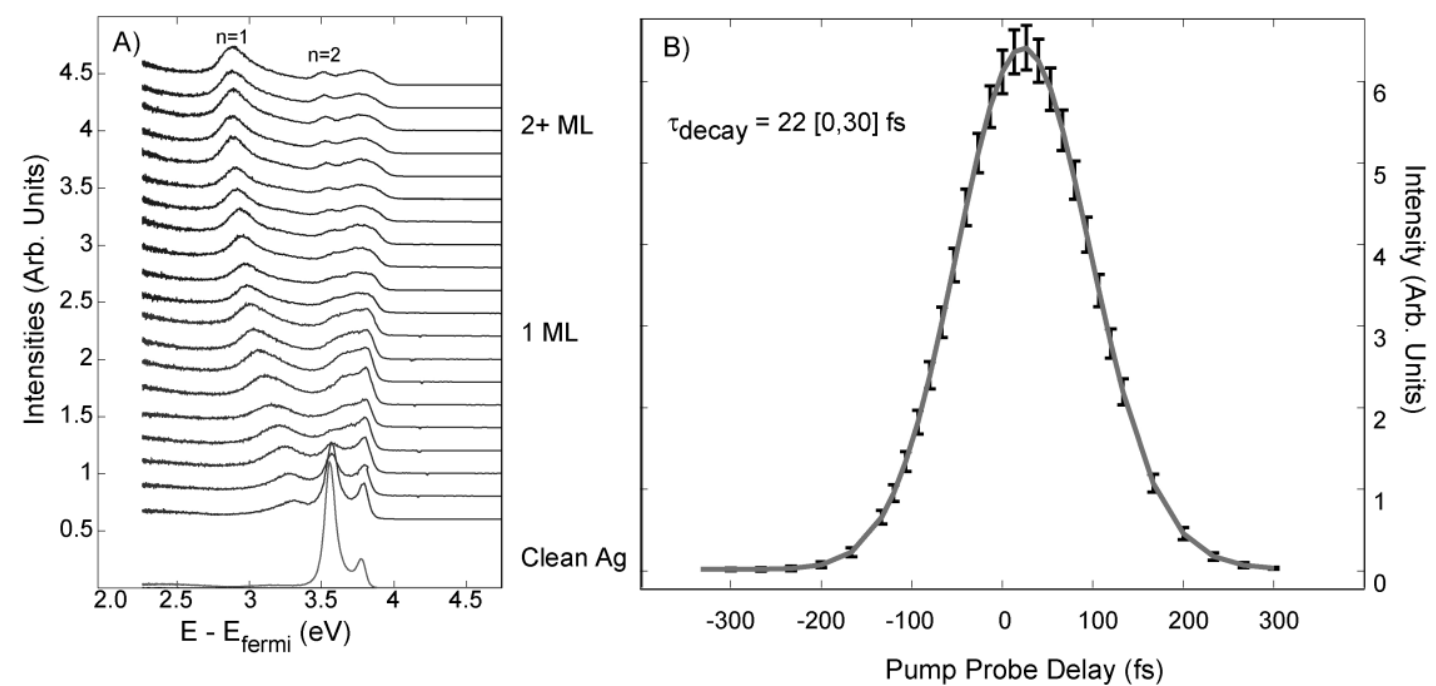

Figure 4.2 : A) Two Photon Photoemission of $6 \mathrm{~T}$ lying flat on $\mathrm{Ag}(111)$ of increasing thicknesses. The two states seen are the $n=1$ and $n=2$ image potential states. B) Dynamics of the $n=1$ IPS for one monolayer. The decay time for all thicknesses remains under $30 \mathrm{fs}$.

For DH6T adsorbed on $\operatorname{Ag}(111)$, we see a work function shift of $-0.74 \pm 0.06 \mathrm{eV}$ compared to the clean silver. There are two states in the $2 \mathrm{PPE}$ spectrum for the mono and bilayer regimes where the aromatic rings lay parallel to the surface shown in figure 3. These states are energetically high lying states at $3.03 \pm 0.06 \mathrm{eV}$ and $3.58 \pm 0.06 \mathrm{eV}$ above the Fermi level, energetically track with the work function shift, and are also assigned as image potential states. The binding energy of the $n=1$ IPS for DH6T $(-0.86$ $\mathrm{eV}$ ) is similar to that of the 6T. These electrons also decay rapidly with a lifetime less than $30 \mathrm{fs}$. This implies that while energetically the electron is sampling over a wide range, it is decaying via the most direct route to the metal surface which is through the aromatic cores, thus giving it the same lifetime as pure 6T/Ag(111). For the lying layers of DH6T, the IPS electron shows a slight shift in energy of $-0.015 \mathrm{eV}$ over its lifetime and no change in its localization. This is due to the efficient coupling of the molecule to the metal via its aromatic core, resulting in ultrashort ( $<30 \mathrm{fs})$ lifetimes for the IPS on flat DH6T. 


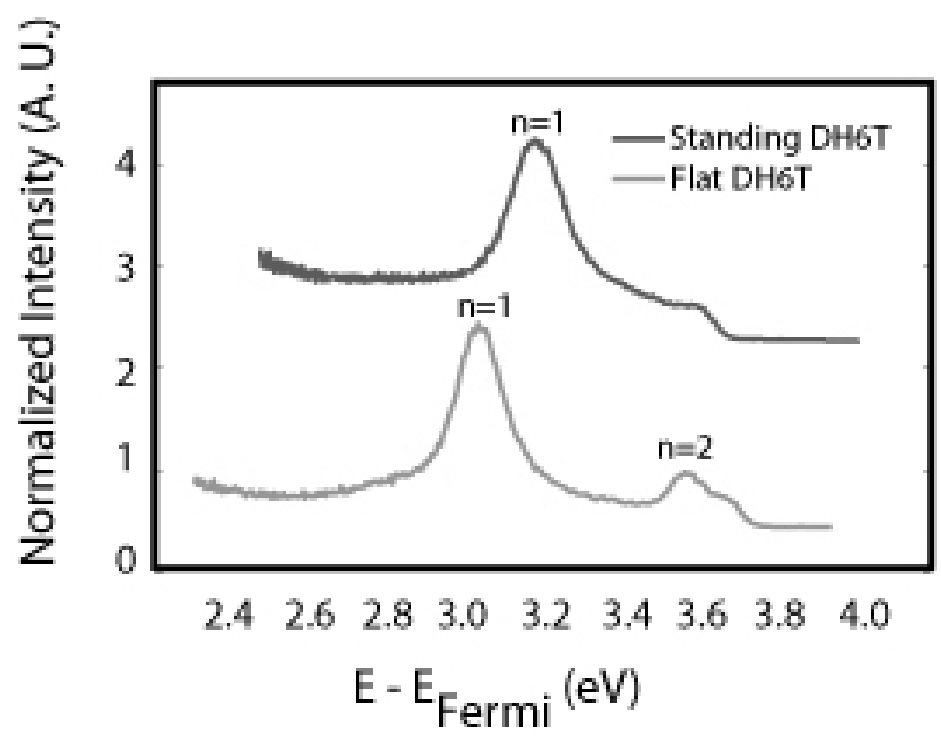

Figure 4.3 Two Photon Photoemission spectrum of Flat (bottom spectrum) and Standing (top spectrum) DH6T / Ag(1110

For thicker coverages (> $2 \mathrm{ML}$ ), DH6T tilts upright to a vertical configuration, and sits with the molecular axis at an angle of $28^{\circ}$ to the surface normal ${ }^{62}$. For these thicker films, the binding energy of the IPS electron at t0 is also decreased by $100 \mathrm{meV}$. The lifetime of the IPS is increased by a factor of 3 to $80 \mathrm{fs} \pm 20 \mathrm{fs}$. Furthermore, the energy of the IPS at the bottom of the band decreases by $80 \mathrm{meV}$ over time for the multilayer. This is illustrated in Figure 4.4. At no point in the spectrum are there two separate peaks, indicative of charge transfer or a defect based trap state. Instead, the center energy of the IPS state decreases linearly with time at a rate of $20 \mathrm{meV} / 100 \mathrm{fs}$.

Concurrent with the energy relaxation of the IPS state, the IPS changes from a delocalized to a localized electron. By varying the angle of the sample with respect to the detector, we allow only specific combinations of energy and momentum photoelectrons to contribute to the signal. The relationship between the momentum of the electron and its energy and detection angle is given in equation 1.

$$
k_{/ /}=\sin \theta \frac{\sqrt{2 m_{e} E_{k i n}}}{\hbar}
$$

By changing this angle, 2PPE can then map out the excited state band structure of a material. At each time slice, we can measure the intensity of the IPS electron as a function of both $\mathrm{k}_{/ /}$and energy. For the bare $\operatorname{Ag}(111)$ surface, the IPS is nearly free electron like and has an effective mass of $1.3 \mathrm{~m}_{e}$, where $\mathrm{m}_{\mathrm{e}}$ is the mass of a bare electron. For dielectrically screened IPS electrons, the effective mass ranges between 1 and 1.5. Using simple band transport theory, this corresponds to a lower bound on the charge mobility of $30 \mathrm{~cm}^{2} / \mathrm{Vs}$. For systems in which the nuclear motions of the molecule interact strongly with the IPS, the effective mass of the electron can grow, leading to a small polaron state $\left(\mathrm{m}^{*} \rightarrow \infty\right)$. 


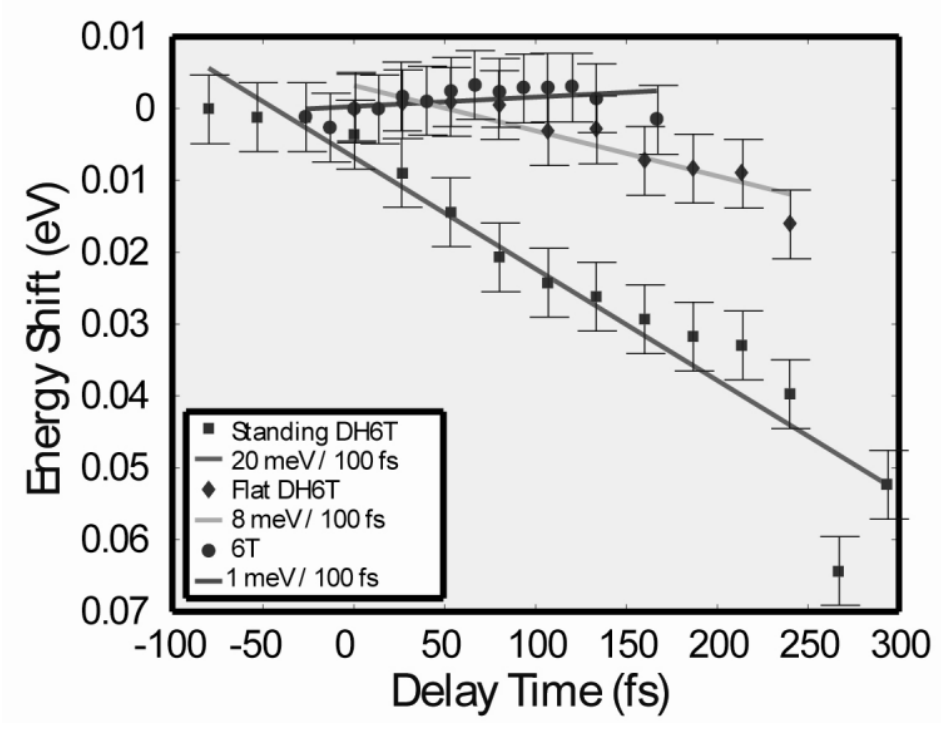

Figure 4.4 Energy shift of the $n=1$ IPS for all the three films from its $t=0$ value.

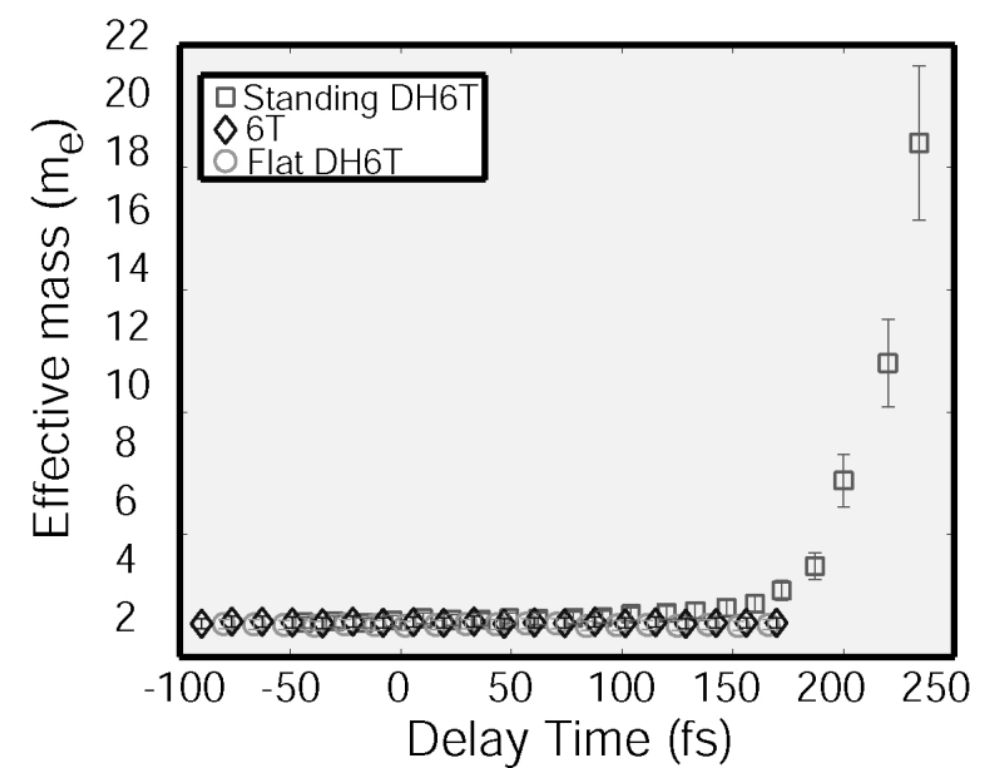

Figure 4.5 Effective mass for all 3 films as a function of time. Only the standing DH6T shows evidence of electron localization.

In Figure 4.5 we show the effective mass of the IPS electron for both DH6T and $\alpha$-sexithiophene on $\operatorname{Ag}(111)$ over time. For all of the morphologies except standing DH6T, the effective mass stays between 1.2 and $1.5 \mathrm{~m}_{\mathrm{e}}$. For the thicker coverages of DH6T, the effective mass starts at $1.3 \mathrm{~m}_{\mathrm{e}}$, and increases to $21 \pm 0.5 \mathrm{~m}_{\mathrm{e}}$ by $233 \mathrm{fs}$. This signifies that charges which are initially delocalized over the entire surface are collapsing 
to point charges which are incapable of lateral movement without resorting to thermal hopping. By Fourier transforming the spectral intensity vs. $\mathrm{k}_{/ /}$we can determine the spatial extent of the localized state. Using the rule of thumb presented by Bezel at al. in equation 2 the size of the final state is estimated at $14 \pm 4 \AA$ FWHM, which is the same size as the intermolecular spacing between two adjacent DH6T molecules ${ }^{65}$.

$$
\Delta x_{F W H M}=\frac{4 \ln (2)}{\Delta p_{F W H M} / \hbar}
$$

The error in determining the effective masses was difficult to quantify because it comes from two components, the uncertainty in the angle of the sample $\left( \pm 1^{\circ}\right)$ and the uncertainty in our kinetic energy axis $( \pm 0.005-0.015 \mathrm{eV})$. These two parameters are coupled into the uncertainty in the $\mathrm{k}_{/ /}$determination. To determine the inherent error in the measurement, random distributions of energy and $\mathrm{k}_{/ /}$were created with the errors mentioned above centered about the positions which give the experimentally measured effective masses. Two times the standard deviation of this distribution for each effective mass was used to determine the error. This error still seems low given variation in sample preparation, therefore the error bars used were $\pm 15 \%$ of the measured effective mass, which is roughly four times $\pm 4 \sigma$ of the above distribution

\subsection{Discussion}

There are three possible explanations for the existence of a dynamically localizing electron in the standing DH6T layers and nowhere else: IPS - molecular orbital coupling, trap sites, or small polaron formation. By changing the orientation of the conjugated axis relative to the surface, there could be an increase in the direct overlap between the IPS wave function and unoccupied electronic states of the molecule. However, in order for the electron to interact with unoccupied states of the standing portion of the DH6T layer, the electron would need to be on far side of the standing hexyl groups from the metal surface. These hexyl groups would then present an energetic barrier to the electron through which it would need to tunnel in order to return to the metal and relax thus increasing the electron's lifetime into the picosecond regime. This lack of lifetime enhancement means that the electron is still close to the metal surface, and therefore not strongly interacting with the electronic states of the standing aromatic core of the multilayer.

Distinguishing between an electron trapped into a defect site and a self localizing small polaron is more difficult because of the ultrafast lifetime of $80 \pm 20 \mathrm{fs}$. Previous determinations of small polaron formation verified the mechanism by comparing the relaxation rates of the delocalized electrons at differing parallel momentum ${ }^{39}$. After subtracting a relaxation rate for tunneling back to the metal, which was assumed to be momentum independent, these rates were then fit to a quantum mechanically modified Marcus electron transfer theory which has been shown to be isomorphic with Holstein polaron formation. Unfortunately, in this system the electronic decay rate is too fast to be able to perform such an analysis with confidence. 
Studying the decay rate versus the sample temperature led to a negligible change in the decay rate $(69 \pm 20 \mathrm{fs}$ at $305 \mathrm{~K}$ vs. $80 \pm 20 \mathrm{fs})$. Changing the temperature did, however, significantly change the intensity of background scattering for photo-electrons. The large temperature dependence to the inelastic scattering suggests that the vibrations of the DH6T rather than defects are the primary mechanism for surface scattering of the excited electrons. Thiophene based oligomers also typically grow in large domains without defects when evaporated onto a single crystal surface ${ }^{66,67}$. Furthermore, there is no point in time in which there exists both a delocalized and localized peak, as one would expect for electrons being trapped by encountering defects. More recent work on 2D small polaron formation by $\mathrm{Ku}$ and Trugman suggest that the dynamics of small polaron formation can happen on a time scale as fast as 5 vibrational periods for states created with low initial momentum ${ }^{68,69}$. The vibration previously identified as highly coupled to polaron formation in alkyls is the methyl rocking motion at $750 \mathrm{~cm}^{-1}$ which corresponds to a period of $44 \mathrm{fs}$. Five vibrational periods would be $220 \mathrm{fs}$, which is the same timescale that we see for localization of the IPS. We thus suspect that the state is being localized by alkyl groups, though we cannot definitively rule out the possibility of charge trapping.

Regardless of the exact nature of the of the state, whether it is vibrationally self induced or exists due to defects in packing, it still represents an upper bound for the mobility of an electron traveling through DH6T. The morphologies of both the 6T and DH6T layers on $\operatorname{Ag}(111)$ are known and exhibit long range order. The electron being excited is being excited directly into a delocalized state, thus eliminating the possibility that the excitation is inducing some intramolecular torsion as can happen with a direct HOMO to LUMO transition. For the sexithiophene systems, although the lifetime is short due to proximity to the metal surface, there is no evidence of any electronic localization. In comparison, for multilayers of DH6T, we see a strong and continuous mass enhancement of the excited electron, which eventually becomes a fully localized state with the same lateral size of a single DH6T molecule. Furthermore, the energy of the localized state is stabilized by over $50 \mathrm{meV}$ from the original delocalized state (Figure 4.4).

The implication for charge transport is that if an organic semiconductor and its alkylated counterpart are placed in an environment with a high degree of crystallinity and low rotational disorder, the unsubstituted molecules should make materials with high charge mobilities and better transport characteristics. This is due to the localization of electrons which occurs in the presence of alkanes. Furthermore, these localized electrons are stabilized by over $50 \mathrm{meV}$ relative to their delocalized counterparts. This loss of energy would show up as wasted heat, and would not be recoverable for work. Although this may seem like a small amount, the net energy loss in these experiment is limited by the ultrafast lifetime of the charges. The $50 \mathrm{meV}$ is the relaxation which occurs in just the first couple hundred femtoseconds. Further relaxation is certainly expected though at a slower rate. If, however, the environment is disordered enough such that the competitive Van der Waal's interaction between alkyl and aromatic moieties increases the orbital overlap between adjacent molecules, then the alkylated molecules may become the better conductor of charge, but it will not be able to approach the currents and 
charge mobilities of inorganic systems because charges will be trapped the alkyl components of the system.

In conclusion, we have presented angle and time resolved two photo-emission studies of the image potential states for both 6T and DH6T on $\operatorname{Ag}(111)$. For 6T and DH6T lying parallel to the surface, the electron remains delocalized over its lifetime. For more than 2ML of DH6T when the molecules rotate to point their hexyl groups at the metals surface, the IPS electrons were found to become tightly localized to $14 \pm 4 \AA$, roughly the spacing between adjacent DH6T molecules on the surface. Based on the lifetime of these states, the localized state was determined not to be a purely electronic phenomenon, and results either from small polaron formation or trapping based on the morphology of the layer. The implications for device performance based on the charge localizing nature of alkyl groups were discussed. 


\section{Chapter 5}

\section{$\operatorname{Mg} / \operatorname{Ag}(111)$}

\subsection{Introduction}

Interfacial and image potential states at bimetallic interfaces has a received a great deal of attention in the last several years. Part of the motivation for studying these interfaces is fundamental. Ultrathin films of high work function transition metals have been deposited onto lower work function noble metals by Osgood to make interfacial quantum well states. The energy and lifetimes of the lifetimes of charge transfer states from the ns orbital of alkali metals into copper and silver have been studied as a model for the simplest possible chemisorbtive potentials. Part of the motivation has been more applications driven. The interface between two metals can be used as a modeling system for studying the electronic environment of core-shell nanoparticles of differing metals. Metal surfaces can also be used as a geometric template for growing new metals and metal oxide layers with new lattice constants to get novel thermal and electronic properties.

The goals in studying the $\mathrm{Mg} / \mathrm{Ag}$ interface were two fold. The first goal was to study it as a stepping stone for creating the $\mathrm{MgO}(111) / \mathrm{Ag}(111)$ interface. $\mathrm{MgO}$ has been heavily studied for its potential use as a water splitting electrode ${ }^{70}$, and as a potential high-k dielectric for replacing silicon oxide and silicon carbide as a gate in transistors ${ }^{71}$, 72 . Typically, metal oxides grown on metal surfaces are grown in the $\{100\}$ directional family, and only recently have the (111) faces been studied. The reason is that for salts in the halite crystal structure such as $\mathrm{MgO}$ or alkali halides, the (111) face consists of alternating planes consisting of only cations or anions. The surface energy of creating such a uniformly charged surface is prohibitive, and these surfaces are unstable unless they are under vacuum, or grown on top of a conducting substrate with which they can exchange some charge.

Recent work by two groups suggest that $\mathrm{MgO} / \mathrm{Ag}(111)$ should be possible. Kiguchi et al. dosed $\mathrm{Mg}$ onto $\mathrm{Ag}(111)$ in an oxygen rich atmosphere, and used a combination of reflection high energy electron diffraction (RHEED) and ultraviolet photoemission spectroscopy (UPS) to determine a stable $\mathrm{MgO}(111) / \mathrm{Ag}(111)$ interface $^{73}$,

74. Goniakowski et al. used ab initio electronic structure calculations to determine that a more likely geometry for the results observed by Kiguchi was the (0001) face of a graphite structure, rather than any face of the typical rock salt structure ${ }^{75}$. 
The more fundamental reason for investigating the $\mathrm{Mg} / \mathrm{Ag}(111)$ interface was to investigate the surface band gap at that interface using the IPS as a probe. At a surface, the bulk periodic potential is abruptly broken in one direction. For most transition metals and the noble metals, this results in a large band gap opening up at the surface somewhere between the Fermi level and the vacuum. The natural linewidth of most image potential states is limited by lifetime broadening, which is determined by the energetic positioning of the vacuum level (and the image potential states which are pinned to it) relative to the surface band gap $^{2}$. Image potential states which lie in the gap have long lifetimes, others do not. For magnesium the (0001), the surface band gap does not lie between the Fermi level and the vacuum, but rather over $1.8 \mathrm{eV}$ below the Fermi level. Chulkov and Silkin performed ab initio GW calculations of the surface band structure, and found no accumulation in the surface projected density of states for the IPS. This makes magnesium unique among metals studied so far, because even other metals whose IPS does not fall in a band gap still show a Fano style resonance of a discrete IPS coupled to the bulk continuum. A metal surface with no bound IPS could be useful as a charge injection electrode for devices, since charges have been shown to scatter and be trapped off of the IPS at other metal electrodes.

\subsection{Experimental}

99.995\% Magnesium was purchased from Sigma-Aldrich in 50 mesh powdered from, meaning that the particle diameters were under $300 \mu \mathrm{m}$. The powdered was then placed into the crucible of a Knudsen cell and degassed over night. Magnesium was dosed by heating the effusion cell up to $623 \mathrm{~K}$ which results in dosing at a flux that equaled roughly 1 monolayer per 6 minutes of exposure. Because the surface of Magnesium oxidizes very easily, there were contamination concerns about dosing the magnesium from a powdered source rather than from a single metal ribbon. Therefore a 25 mesh Magnesium (particle size $<710 \mu \mathrm{m}$ ) and shaved Magnesium turnings were also used. The 25 mesh powder showed similar results to the 50 mesh. The turnings, however, suffered from poor thermal contact with the crucible, and did not reproducibly provide the same flux at a given temperature over multiple experiments.

\subsection{Results}

The growth of $\mathrm{Mg}$ on the $\mathrm{Ag}(111)$ surface is not reported elsewhere in the literature, although the growth of $\mathrm{Mg}$ on $\mathrm{Ag}(100)$ has been ${ }^{76}$. Here, Auger spectroscopy and LEED were used to characterize the growth of $\mathrm{Mg} / \mathrm{Ag}(111)$. Two peaks were visible in the Auger spectrum for Magnesium on at $46 \mathrm{eV}$ and one at $34-36 \mathrm{eV}$, as shown in figure 5.1. As the coverage of $\mathrm{Mg}$ increased, the low energy peak shifted to higher energies and its position became dependent on the energy and intensity of the electron beam used. Therefore it was not used to characterize the growth mode. The doublet of lines for silver at 351 and $358 \mathrm{eV}$ were used to monitor film growth.

Shown in figures 5.2 and 5.3 are the intensity of the primary $\mathrm{Ag}$ and $\mathrm{Mg}$ peaks as a function of dosing time for $\mathrm{Mg}$ on to $\mathrm{Ag}(111)$ in 60 second intervals at two different 


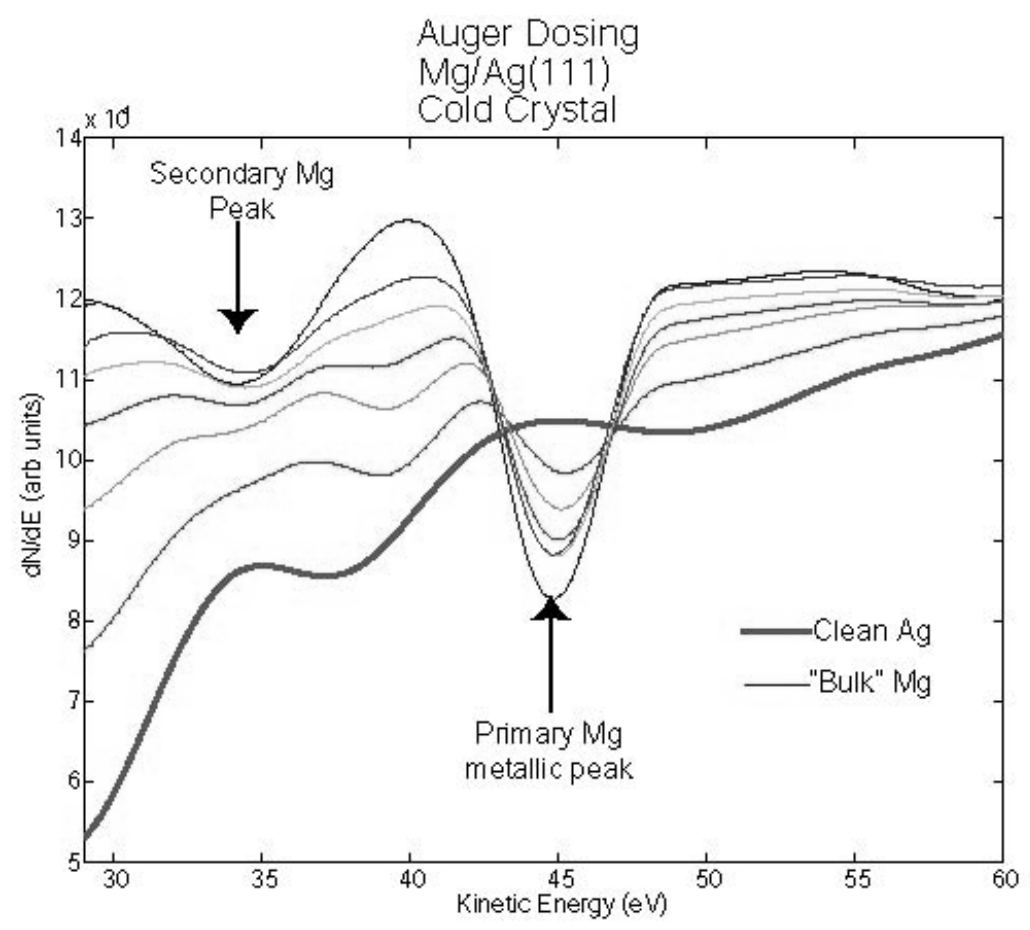

Figure 5.1 Auger Spectrum of the low energy region of $\mathrm{Mg}$ dosed on a $\mathrm{Ag}(111)$ crystal below150 $\mathrm{K}$.

temperatures. At room temperature, the $\mathrm{Mg}$ peak saturates exponentially with a time constant of 550 seconds, and the Ag peak exponentially decays with a time constant of 400 seconds. When $\mathrm{Mg}$ is dosed at cryogenic temperatures $(\mathrm{T}<150 \mathrm{~K})$, the $\mathrm{Ag}$ peaks still decay exponentially, but with a faster time constant of 250 seconds. The rise of the $\mathrm{Mg}$ peaks, however, is not consistent with exponential behavior.

LEED of $\mathrm{Mg} / \mathrm{Ag}(111)$ was also taken at sub ML and multilayers coverages dosed at room temperature then cooled. Sample images are shown in Figure 5.4. Based on the difference between the scattering angle for the Mg and Ag peaks, the nearest neighbor distance for magnesium atoms grown on the surface is $3.18 \AA$, just slightly shorter than the $3.21 \AA$ lattice constant for $\mathrm{Mg}$ in the hcp crystal. No spots were seen that indicated that sub monolayer coverages adsorbed in an organized super lattice unlike the $c(2 \times 2)$ lattice observed by Tegencamp et $\mathrm{al}^{76}$. However, since the goal of the experiment was to create clean monolayers and then oxidize them, more diligent efforts in this direction could have been made.

Finally, two photon photoemission spectra were taken of the cold and warm layers of $\mathrm{Mg} / \mathrm{Ag}(111)$. Spectra shown for $1-4 \mathrm{ML}$ equivalents are shown in Figure 5.5. Upon dosing $\mathrm{Mg}$, two peaks appear in the TPPE spectrum. By a single monolayer the peak widths are 250 and $150 \mathrm{meV}$ for the low and high energy peaks respectively. Past one monolayer, the spectra is better fit by three peaks which continue to shift and broaden with increasing coverage. Past four monolayer equivalents, the peaks become too broad to be able to fit, and the spectrum is essentially featureless. 

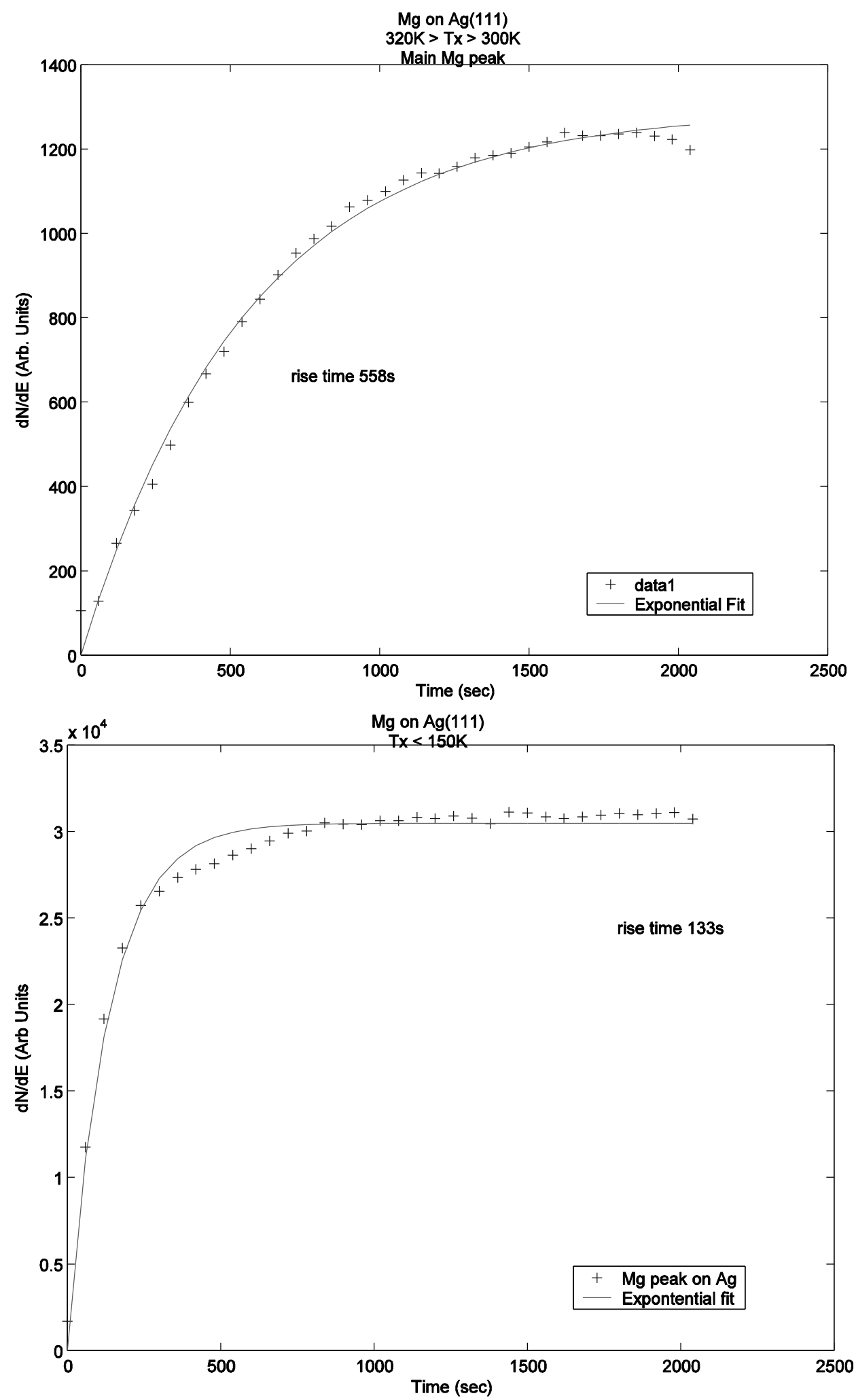

Figure 5.2 Top panel shows the intensity of the $\mathrm{Mg}$ Auger peak at $46 \mathrm{eV}$ for increasing exposure time of $\mathrm{Mg}$ dosed onto an $\mathrm{Ag}(111)$ substrate near room temperature. Bottom panel shows the same but for a $150 \mathrm{~K} \mathrm{Ag(111)} \mathrm{crystal.}$ 

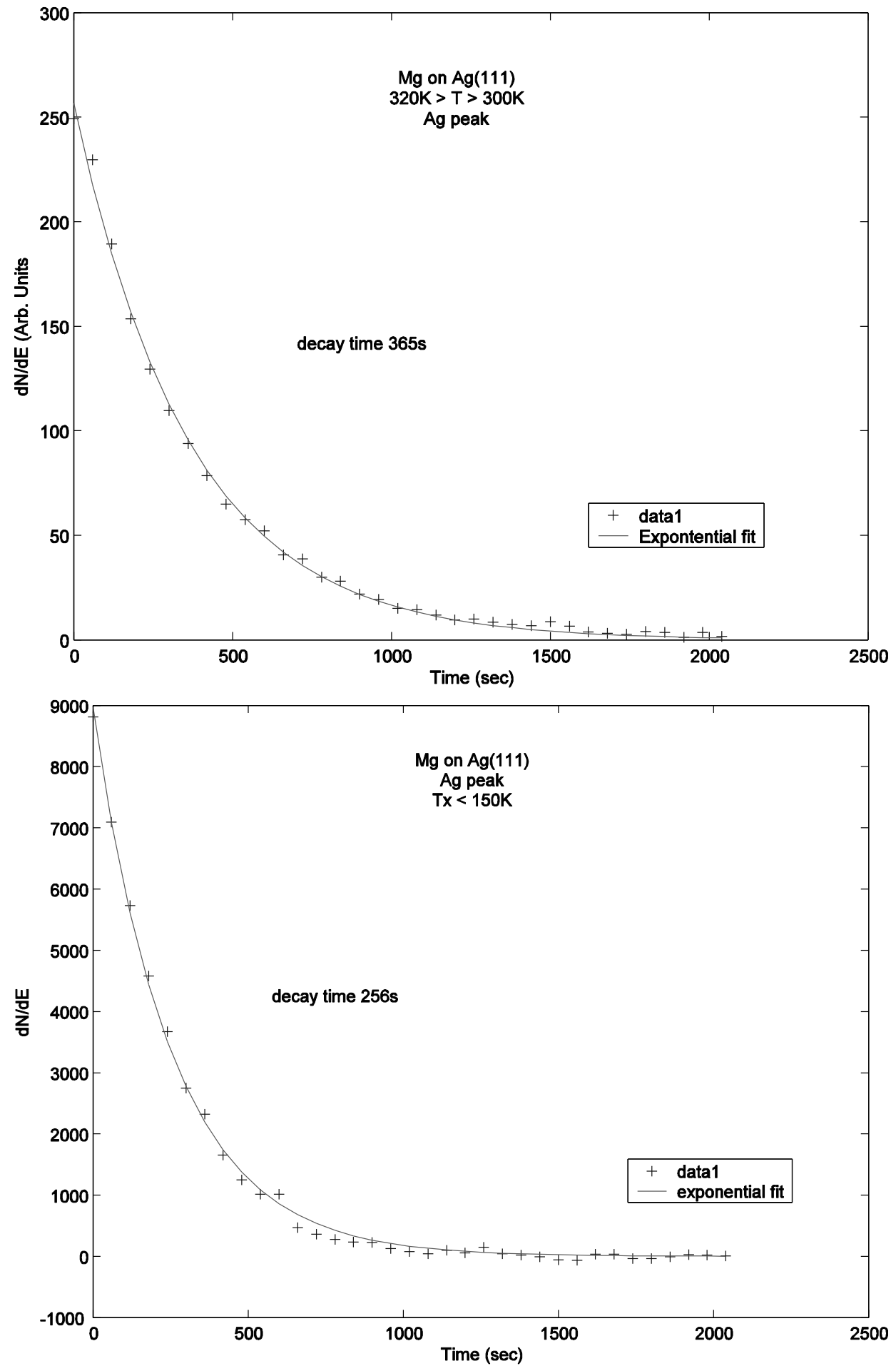

Figure 5.3 Top panel shows the intensity of the Ag Auger peak at $351 \mathrm{eV}$ for increasing exposure time of $\mathrm{Mg}$ dosed onto an $\mathrm{Ag}(111)$ substrate near room temperature. Bottom panel shows the same but for a $150 \mathrm{~K} \mathrm{Ag(111)} \mathrm{crystal.}$ 


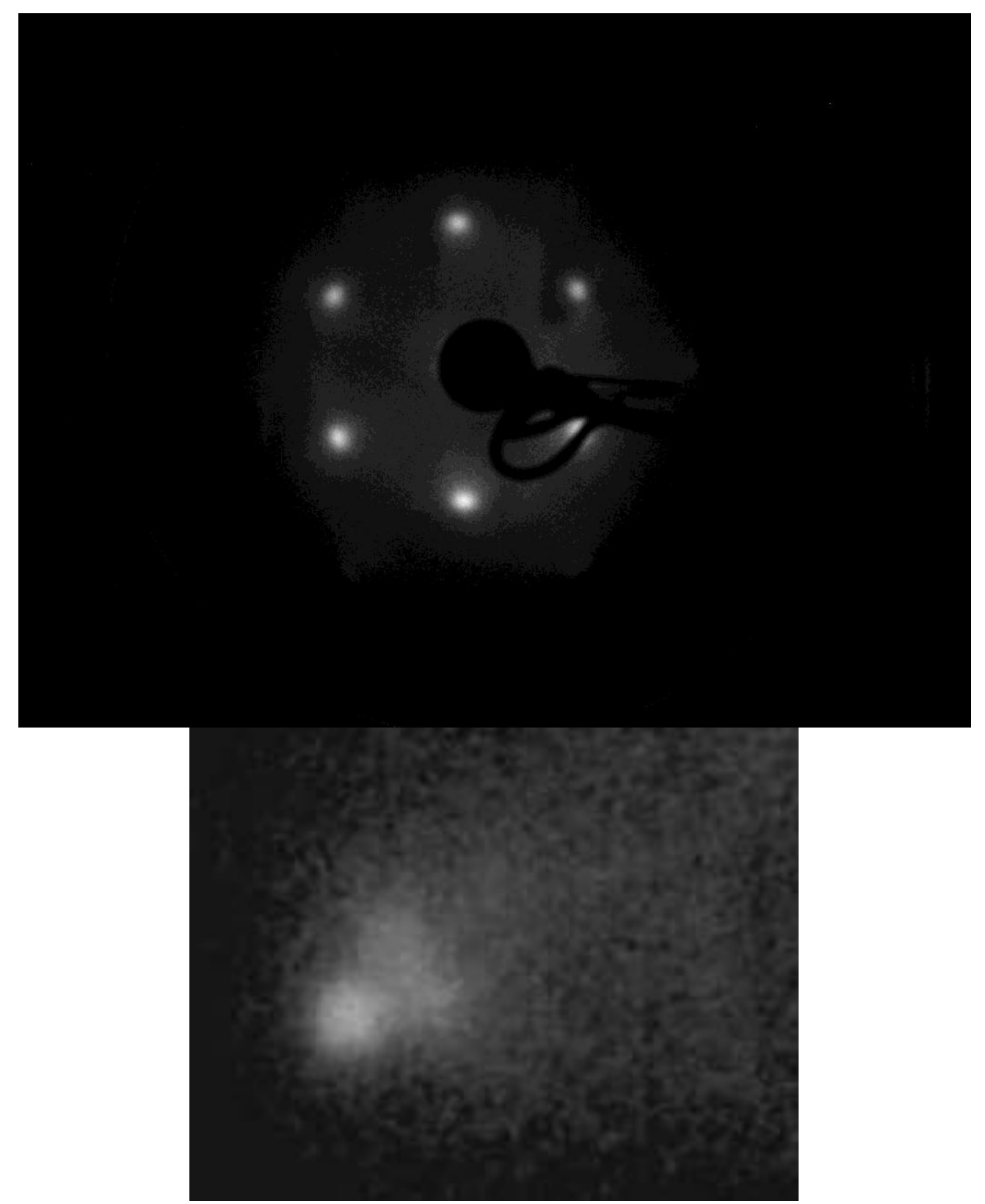

Figure 5.4 Top panel shows LEED images of $\mathrm{Mg} / \mathrm{Ag}(111)$ grown at room temperature. Only hexagonal ordering is observed. The bottom panel shows a close-up image of a single diffraction spot after $300 \mathrm{~s}$ of dosing such that spots for both the $\mathrm{Ag}(\mathbf{1 1 1})$ (the spot closest to bottom left corner) and $\mathrm{Mg}$ adsorbate can be seen. 


\section{Multilayer Coverages of $\mathrm{Mg} / \mathrm{Ag}(111)$}

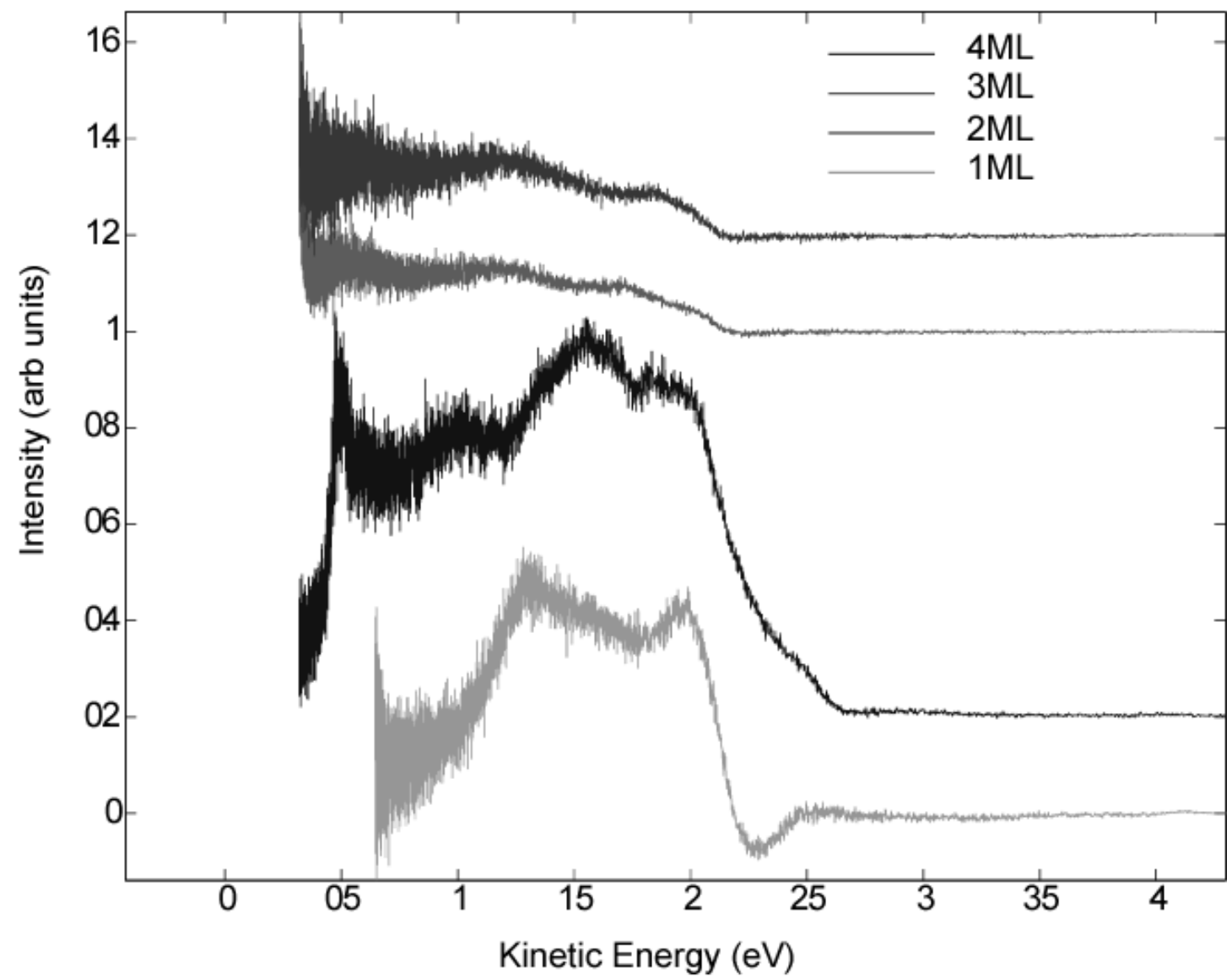

Figure 5.5 Two photon photoemission spectra for differing coverages of $\mathrm{Mg} / \mathrm{Ag}(111)$. Samples were grown while keeping the $\mathrm{Ag}(111)$ substrate under $150 \mathrm{~K}$. Coverage is highest for the top spectra and lowest for the bottom.

\subsection{Discussion}

Auger Spectroscopy and X-ray photoemission have both been used to distinguish between island formation and layer by layer growth of epitaxially deposited adsorbates ${ }^{30}$, 76. The concept is that electrons from each layer on the surface a probability of escaping the surface, and that probability depends on how many layers are between it and vacuum. Deeply buried atoms contribute negligibly to the XPS and Auger signals, while atoms at the vacuum interface produce a strong signal. When layer by layer growth occurs, the signal due to the adsorbate increases linearly with coverage until a complete monolayer is formed. The signal continues to rise linearly with the addition of a second monolayer, but with a shallower slope. This process of linear signal increases with abrupt changes in slope continues until additional layers increase the thickness of the film beyond the signal electron's escape depth, and the signal saturates. When the adsorbates form islands rather than neat layers, each island has a distribution of atoms each level of thickness. This distribution usually follows passion statistics, and experimentally results in a signal strength versus exposure time that fits well to an exponential. 
A transition between these two growth modes as a function of temperature appears to happen in the $\mathrm{Mg} / \mathrm{Ag}(111)$ system. For a $300 \mathrm{~K}$ substrate both the $\mathrm{Mg}$ and the Ag peaks show clear exponential behavior. For a $150 \mathrm{~K}$ substrate, the bottom panel of Figure 5.2 shows two abrupt changes of slope, one after 300 seconds and one after 660700 seconds. Although there is not a clean change in the slope of the silver Auger peaks, the intensity does decay faster than for the room temperature silver, indicating that the surface is being more evenly covered. This is similar to the growth mode transition that Tegenkamp et al. observed for $\mathrm{Mg} / \mathrm{Ag}(100)$ below $200 \mathrm{~K}$.

The most likely source for this transition is strain in the lattice of the $\mathrm{Mg}$. The native structure for Magnesium is an hcp crystal with a lattice constant $11 \%$ larger than the $\operatorname{Ag}(111)$ nearest neighbor distance of $2.89 \AA$. Lattice mismatch creates a strain in the layer which accumulates proportionally to the volume, and competes with the natural tendency of adsorbates to minimize their surface energy by presenting a planar structure. Elevating the temperature increases the ability of surface atoms beyond the outermost layer to diffuse and find the thermodynamic minimum. Further evidence for the layer by layer growth being a kinetic trap is that the transition is not reversible. Layers grown at room temperature and then cooled show the same Auger intensity as the warm layer.

Having found a way to create layered structures of $\mathrm{Mg} / \mathrm{Ag}(111)$, the evolution of the surface band structure was studied using TPPE. Adsorption of the first monolayer shifted the work function to $3.76 \mathrm{eV}$, and adsorption of a second reduced the work function to $3.6 \mathrm{eV}$. Further layers produced no resolvable changes in the work function. Figure 5.4 shows an accelerated TPPE spectrum of 1-4 ML of $\mathrm{Mg} / \mathrm{Ag}(111)$. The spectra needed to be accelerated in order to determine whether the lower energy features were actual peaks or electron scattering events. The feature at $1.35 \mathrm{eV}$ in the $1 \mathrm{ML}$ spectrum is the IPS which shifts smoothly from its clean silver value. The actual binding energy of this state is $-1.05 \mathrm{eV}$ below the vacuum level. The second higher feature in the $1 \mathrm{ML}$ spectrum does not fit to a hydrogenic progression, and remains unassigned. Dosing a single monolayer reduces the lifetime of the $n=1$ IPS from 22 fs on clean $\operatorname{Ag}(111)$ to a value to small to measure with the current setup. Dosing additional Mg splits the $n=1$ IPS into two states. As the coverage increases, the width of each peak increases significantly presumably due to lifetime broadening, and the overall intensity decreases. This is consistent with the prediction of Chulkov et $\mathrm{al}^{77}$ which predict that the lifetime of electrons $1 \mathrm{eV}$ below the vacuum level in $\mathrm{Mg}$ to be $3 \mathrm{fs}$.

Attempts were made to controllably oxidize the $1 \mathrm{ML} / \mathrm{Ag}(111)$ surface in such a way that gave an ordered surface. This was done by introducing $99.998 \%$ oxygen into the chamber after dosing $1 \mathrm{ML} \mathrm{Mg} / \mathrm{Ag}(111)$, by co-dosing $1 \mathrm{ML} / \mathrm{Ag}(111)$ in $1 \times 10^{-6}$ torr $\mathrm{O}_{2}$, and co-dosing $0.5 \mathrm{ML} / \mathrm{Ag}(111)$ in $1 \times 10^{-6}$ torr $\mathrm{O}_{2}$. Annealing at various temperatures between $150 \mathrm{~K}$ and $400 \mathrm{~K}$ for all three systems was attempted. Each system resulted in the uptake of oxygen as determined by the presence of the oxygen KLL Auger peak at $513 \mathrm{eV}$. The work function for each of these systems was also lowered to $3 \mathrm{eV}$ or slightly below. Two photon photoemission spectra were taken by bypassing the OPA, and using $800 \mathrm{~nm}$ and $400 \mathrm{~nm}$ light. At no point were any peaks observed in the two photon spectra. Similarly, no LEED spots were observed once oxygen was dosed into the main chamber. Although this project had the potential to be interesting by shedding light on the electronic structure of a high dielectric insulator at a metal surface, the lack of 
order, spectral features, and inability to assign the $\mathrm{Mg} / \mathrm{Ag}(111)$ spectra resulted in the project being abandoned. 


\section{References}

1. Jackson, D., Classical Electrodynamics. 3rd ed.; Wiley: New York, 1999.

2. Echenique, P. M.; Berndt, R.; Chulkov, E. V.; Fauster, T.; Goldmann, A.; Höfer, U., Decay of electronic excitations at metal surfaces. Surface Science Reports 2004, 52, (7-8), 219-317.

3. García-González, P.; Alvarellos, J. E.; Chacón, E.; Tarazona, P., Nonlocal WDA functional capable of describing the image potential of a metallic surface. International Journal of Quantum Chemistry 2003, 91, (2), 139-144.

4. White, I. D.; Godby, R. W.; Rieger, M. M.; Needs, R. J., Dynamic Image Potential at an Al(111) Surface. Phys. Rev. Lett. J1 - PRL 1998, 80, (19), 4265 LP - 4268.

5. Grimes, C. C.; Brown, T. R., Direct Spectroscopic Observation of Electrons in ImagePotential States Outside Liquid Helium. Phys. Rev. Lett. J1 - PRL 1974, 32, (6), 280 LP - 283.

6. Steinmann, W., Two-Photon Photoemission Spectroscopy of Electronic States at Metal Surfaces. physica status solidi (b) 1995, 192, (2), 339-356.

7. Lindstrom, C. D.; Muntwiler, M.; Zhu, X. Y., Electron dynamics at polyacene/Au(111) interfaces. Journal of Physical Chemistry B 2007, 111, 6913-6920.

8. Knoesel, E.; Hotzel, A.; Wolf, M., Ultrafast dynamics of hot electrons and holes in copper: Excitation, energy relaxation, and transport effects. Physical Review B 1998, 57, 1281212824.

9. Steinmann, W., Spectroscopy of Image-Potential States by 2-Photon Photoemission. Applied Physics a-Materials Science \& Processing 1989, 49, (4), 365-377.

10. Kutzner, J.; Paucksch, R.; Jabs, C.; Zacharias, H.; Braun, J., High-resolution photoelectron emission spectroscopy of surface states on $\mathrm{Ni}(111)$. Physical Review B 1997, 56, (24), 1600316009.

11. Weinelt, M.; Kutschera, M.; Schmidt, R.; Orth, C.; Fauster, T.; Rohlfing, M., Electronic structure and electron dynamics at Si(100). Applied Physics a-Materials Science \& Processing 2005, 80, 995-1003.

12. Silkin, V. M.; Zhao, J.; Guinea, F.; Chulkov, E. V.; Echenique, P. M.; Petek, H., Image potential states in graphene. Physical Review B 2009, 80, (12).

13. Zamkov, M.; Woody, N.; Bing, S.; Chakraborty, H. S.; Chang, Z.; Thumm, U.; Richard, P., Time-resolved photoimaging of image-potential states in carbon nanotubes. Phys. Rev. Lett. J1 PRL 2004, 93, (15).

14. Fauster, T. a. S., W, Two-photon photoemission spectroscopy of image states. In Electromagnetic Waves: Recent Developments in Research: Photonic Probes of Surfaces Halevi, Ed. Elselvier: Amsterdam, 1995; Vol. 2.

15. Smith, N. V., Phyiscal Review B 1985, 32, 3549.

16. de Andrés, P.; Echenique, P. M.; Flores, F., Lifetime in a two-dimensional imagepotential \& induced electron band. Physical Review B 1987, 35, (9), 4529.

17. U. Hofer, I. L. S., Ch. Reuss, U. Thomann, Th. Fauster, Time-Resolved Coherent Photoelectron Spectroscopy of Quantized Electronic States on Metal Surfaces. Science 1997, 277, (5331), 1480-1482.

18. C.B.Harris, N.-H. G., R. L. Lingle, J. D. McNeil, and C. M. Wong, Femtosecond dynamics of electron localization at interfaces. Annual Review of Physical Chemistry 1997, 48, (711).

19. Cole, M. W.; Cohen, M. H., Phys. Rev. Lett. 1969, 23, (21), 1238. 
20. Cole, M. W., Phys. Rev. 1970, 2, (10), 4239.

21. R. L. Lingle, N.-H. G., R. F. Jordan, J. D. McNeill, C. B. Harris, Femtosecond studies of electron tunneling at metal-dielectric interfaces. Journal of Chemical Physics 1996, 105, 3883.

22. Hotzel, A.; Moos, G.; Ishioka, K.; Wolf, M.; Ertl, G., Femtosecond electron dynamics at adsorbate-metal interfaces and the dielectric continuum model. Applied Physics B-Lasers and Optics 1999, 68, (3), 615-622.

23. Berthold, W.; Rebentrost, F.; Feulner, P.; Hofer, U., Influence of Ar, Kr, and Xe layers on the energies and lifetimes of image-potential states on $\mathrm{Cu}(100)$. Applied Physics a-Materials Science \& Processing 2004, 78, (2), 131-140.

24. K. J. Gaffney, S. H. L., A. D. Miller, P. Szysmanski, C. B. Harris, The adsorbate electron affinity dependence of femtosecond electron dynamics at dielectric/metal interfaces. C.B. Journal of the Chinese Chemical Society 2000, 47, 759.

25. Lindstrom, C. D.; Quinn, D.; Zhu, X. Y., Using image resonances to probe molecular conduction at the n-heptane/Au(111) interface. Journal of Chemical Physics 2005, 122, (12).

26. Gaffney, K. J.; Wong, C. M.; Liu, S. H.; Miller, A. D.; McNeill, J. D.; Harris, C. B., Femtosecond electron dynamics at the benzene/Ag(111) interface. Chemical Physics 2000, 251, (1-3), 99-110.

27. Garrett-Roe, S.; Shipman, S. T.; Szymanski, P.; Strader, M. L.; Yang, A.; Harris, C. B., Ultrafast electron dynamics at metal interfaces: Intraband relaxation of image state electrons as friction. Journal of Physical Chemistry B 2005, 109, (43), 20370-20378.

28. Garrett-Roe, S. Ultrafast Electron Dynamics at Dielectric/Metal Interfaces: Intraband Relaxation of Image Potential State Electrons as Friction and Disorder Induced Electron Localization. Univ. of Cal. Berkeley, Berkeley, 2005.

29. Gundlach, L.; Ernstorfer, R.; Willig, F., Ultrafast interfacial electron transfer from the excited state of anchored molecules into a semiconductor. Progress in Surface Science 2007, 82, (4-6), 355-377.

30. Shipman, S. T.; Garrett-Roe, S.; Szymanski, P.; Yang, A. R.; Strader, M. L.; Harris, C. B., Determination of band curvatures by angle-resolved two-photon photoemission in thin films of C-60 on Ag(111). Journal of Physical Chemistry B 2006, 110, (20), 10002-10010.

31. Yang, A., Steven T. Shipman, Sean Garrett-Roe, James Johns, Matt Strader, Paul Szymanski, Eric Muller, and Charles Harris, Two-photon Photo-emission of Ultrathin Film PTCDA Morphologies on $\mathrm{Ag}(111)$. Journal of Physical Chemistry C 2007, In press.

32. Dutton, G.; Zhu, X. Y., Unoccupied states in C-60 thin films probed by two-photon photoemission. Journal of Physical Chemistry B 2002, 106, (23), 5975-5981.

33. Yang, Q.; Muntwiler, M.; Zhu, X. Y., Charge transfer excitons and image potential states on organic semiconductor surfaces. Physical Review B (Condensed Matter and Materials Physics) 2009, 115214 (8).

34. Chulkov, E. V., Silkin, V. M., Echenique, P. M. , Surface Science Surface Science , 437, 330.

35. Wessendorf, M.; Wiemann, C.; Bauer, M.; Aeschlimann, M.; Schneider, M. A.; Brune, H.; Kern, K., Electronic surface structure of $\mathrm{n}-\mathrm{ML} \mathrm{Ag} / \mathrm{Cu}(111)$ and $\mathrm{Cs} / \mathrm{n}-\mathrm{ML} \mathrm{Ag} / \mathrm{Cu}(111)$ as investigated by 2PPE and STS. Applied Physics a-Materials Science \& Processing 2004, 78, (2), 183-188.

36. Wiesenmayer, M.; Bauer, M.; Mathias, S.; Wessendorf, M.; Chulkov, E. V.; Silkin, V. M.; Borisov, A. G.; Gauyacq, J.-P.; Echenique, P. M.; Aeschlimann, M., Lifetime of an adsorbate excitation modified by a tunable two-dimensional substrate. Physical Review $B$ (Condensed Matter and Materials Physics) 2008, 78, (24), 245410. 
37. Chandler, D., and Leung, K., Excess electrons in liquids: Geometrical Perspectives. Annual Review of Physical Chemistry 1994, 45.

38. Bovensiepen, U.; Gahl, C.; Stal`hler, J.; Bockstedte, M.; Meyer, M.; Baletto, F.; Scandolo, S.; Zhu, X. Y.; Rubio, A.; Wolf, M., A Dynamic Landscape from Femtoseconds to Minutes for Excess Electrons at Iceâ^’Metal Interfaces. The Journal of Physical Chemistry C 2008, 113, (3), 979-988.

39. Ge, N. H.; Wong, C. M.; Lingle, R. L.; McNeill, J. D.; Gaffney, K. J.; Harris, C. B., Femtosecond dynamics of electron localization at interfaces. Science 1998, 279, (5348), 202-205.

40. Liu, S. H.; Miller, A. D.; Gaffney, K. J.; Szymanski, P.; Garrett-Roe, S.; Bezel, I.; Harris, C. B., Direct observation of two-dimensional electron solvation at alcohol/Ag(111) interfaces. Journal of Physical Chemistry B 2002, 106, (50), 12908-12915.

41. Szymanski, P.; Garrett-Roe, S.; Harris, C. B., Time- and angle-resolved two-photon photoemission studies of electron localization and solvation at interfaces. Progress in Surface Science 2005, 78, (1), 1-39.

42. Merry, W. R. Image Potential States at Dielectric-Metal Interfaces. Univ. of Cal. Berkeley, 1992.

43. McNeill, J. D. Ultrafast Dynamics of electrons at Interfaces. Univ. of Cal. Berkeley, Berkeley, 1999.

44. Gundlach, L.; Ernstorfer, R.; Riedle, E.; Eichberger, R.; Willig, F., Femtosecond twophoton photoemission at $150 \mathrm{kHz}$ utilizing two noncollinear optical parametric amplifiers for measuring ultrafast electron dynamics. Applied Physics B-Lasers and Optics 2005, 80, (6), 727731.

45. E. W. Castner, M. M., G. Flemming, Journal of Chemical Physics 1987, 86.

46. E. W. Castner, G. F., Bagchi B., Journal of Chemical Physics 1988, 89, 3519.

47. Hotzel, A. W., M.; Gauyacq, J. P., Journal of Physical Chemistry B 2000, 104.

48. Bagchi, B.; Fleming, G. R., DYNAMICS OF ACTIVATIONLESS REACTIONS IN SOLUTION. J. Phys. Chem. 1990, 94, (1), 9-20.

49. Poornimadevi, C. S.; Bagchi, B., Dynamics of a barrierless reaction on a two-dimensional potential surface in solution. Chemical Physics Letters 1990, 168, (3-4), 276-282.

50. Wong, C. M.; McNeill, J. D.; Gaffney, K. J.; Ge, N. H.; Miller, A. D.; Liu, S. H.; Harris, C. B., Femtosecond studies of electron dynamics at dielectric-metal interfaces. Journal of Physical Chemistry B 1999, 103, (2), 282-292.

51. Berthold, W.; Hofer, U.; Feulner, P.; Chulkov, E. V.; Silkin, V. M.; Echenique, P. M., Momentum-resolved lifetimes of image-potential states on $\mathrm{Cu}(100)$. Physical Review Letters 2002, 88, (5), 4.

52. Kim, J. Y.; Lee, K.; Coates, N. E.; Moses, D.; Nguyen, T. Q.; Dante, M.; Heeger, A. J., Efficient tandem polymer solar cells fabricated by all-solution processing. Science $\mathbf{2 0 0 7 , 3 1 7 ,}$ (5835), 222-225.

53. Berkebile, S.; Koller, G.; Puschnig, P.; Ambrosch-Draxl, C.; Netzer, F.; Ramsey, M., Angleresolved photoemission of chain-like molecules: the electronic band structure of sexithiophene and sexiphenyl. Applied Physics A: Materials Science \& Processing 2009, 101-5.

54. Puschnig, P.; Berkebile, S.; Fleming, A. J.; Koller, G.; Emtsev, K.; Seyller, T.; Riley, J. D.; Ambrosch-Draxl, C.; Netzer, F. P.; Ramsey, M. G., Reconstruction of Molecular Orbital Densities from Photoemission Data. Science 2009, 326, (5953), 702-706.

55. Hwang, I. W.; Soci, C.; Moses, D.; Zhu, Z. G.; Waller, D.; Gaudiana, R.; Brabec, C. J.; Heeger, A. J., Ultrafast electron transfer and decay dynamics in a small band gap bulk heterojunction material. Advanced Materials 2007, 19, (17), 2307-+. 
56. Waldauf, C.; Scharber, M. C.; Schilinsky, P.; Hauch, J. A.; Brabec, C. J., Physics of organic bulk heterojunction devices for photovoltaic applications. Journal of Applied Physics 2006, 99, (10).

57. Kline, R. J.; McGehee, M. D., Morphology and Charge Transport in Conjugated Polymers. Polymer Reviews 2006, 46, (1), 27 - 45.

58. Yang, X. N.; Loos, J.; Veenstra, S. C.; Verhees, W. J. H.; Wienk, M. M.; Kroon, J. M.; Michels, M. A. J.; Janssen, R. A. J., Nanoscale morphology of high-performance polymer solar cells. Nano Letters 2005, 5, (4), 579-583.

59. Yang, F.; Shtein, M.; Forrest, S. R., Controlled growth of a molecular bulk heterojunction photovoltaic cell. Nature Materials 2005, 4, (1), 37-41.

60. Rand, B. P.; Genoe, J.; Heremans, P.; Poortmans, J., Solar cells utilizing small molecular weight organic semiconductors. Progress in Photovoltaics 2007, 15, (8), 659-676.

61. Duhm, S.; Heimel, G.; Salzmann, I.; Glowatzki, H.; Johnson, R. L.; Vollmer, A.; Rabe, J. P.; Koch, N., Orientation-dependent ionization energies and interface dipoles in ordered molecular assemblies. Nature Materials 2008, 7, (4), 326-332.

62. Duhm, S.; Salzmann, I.; Koch, N.; Fukagawa, H.; Kataoka, T.; Hosoumi, S.; Nebashi, K.; Kera, S.; Ueno, N., Vacuum sublimed alpha,omega-dihexylsexithiophene thin films: Correlating electronic structure and molecular orientation. Journal of Applied Physics 2008, 104, (3).

63. Yang, A., Steven T. Shipman, Sean Garrett-Roe, James Johns, Matt Strader, Paul Szymanski, Eric Muller, and Charles Harris, Two-photon Photo-emission of Ultrathin Film PTCDA Morphologies on Ag(111). Journal of Physical Chemistry C 2008, 112, 2506-2513.

64. Dutton, G.; Quinn, D. P.; Lindstrom, C. D.; Zhu, X. Y., Exciton dynamics at molecule-metal interfaces: C-60/Au(111). Physical Review B 2005, 72, (4).

65. Bezel, I.; Gaffney, K. J.; Garrett-Roe, S.; Liu, S. H.; Miller, A. D.; Szymanski, P.; Harris, C. B., Measurement and dynamics of the spatial distribution of an electron localized at a metaldielectric interface. Journal of Chemical Physics 2004, 120, (2), 845-856.

66. M. E. Hajlaoui, F. G., L. Hassine, F. Kouki, H. Bouchriha, Synthetic Metals 2002, 129.

67. Ratera, I., Nanotechnology. 16 2005, 235.

68. Ku, L. C.; Trugman, S. A., Quantum dynamics of polaron formation. Physical Review $B$ 2007, 75, (1).

69. Ku, L. C.; Trugman, S. A.; Bonca, J., Dimensionality effects on the Holstein polaron. Physical Review B 2002, 65, (17).

70. Ciston, J.; Subramanian, A.; Marks, L. D., Water-driven structural evolution of the polar MgO (111) surface: An integrated experimental and theoretical approach. Physical Review $B$ 2009, 79, (8).

71. Brennan, B.; McDonnell, S.; Hughes, G., Photoemission studies of the initial interface formation of ultrathin $\mathrm{MgO}$ dielectric layers on the Si(111) surface. Thin Solid Films 518, (8), 1980-1984.

72. Posadas, A.; Walker, F. J.; Ahn, C. H.; Goodrich, T. L.; Cai, Z.; Ziemer, K. S., Epitaxial MgO as an alternative gate dielectric for SiC transistor applications. Applied Physics Letters 2008, 92, (23).

73. Kiguchi, M.; Entani, S.; Saiki, K.; Goto, T.; Koma, A., Atomic and electronic structure of an unreconstructed polar $\mathrm{MgO}(111)$ thin film on $\mathrm{Ag}(111)$. Physical Review B 2003, 68, (11).

74. Arita, R.; Tanida, Y.; Entani, S.; Kiguchi, M.; Saiki, K.; Aoki, H., Polar surface engineering in ultrathin $\mathrm{MgO}(111) / \mathrm{Ag}(111)$ : Possibility of a metal-insulator transition and magnetism. Physical Review B 2004, 69, (23). 
75. Goniakowski, J.; Noguera, C.; Giordano, L., Using polarity for engineering oxide nanostructures: structural phase diagram in free and supported $\mathrm{MgO}(111)$ ultrathin films. Phys. Rev. Lett. J1 - PRL 2004, vol.93, no.21, 215702/1-4.

76. Tegenkamp, C.; Michailov, M.; Wollschläger, J.; Pfnür, H., Growth and surface alloy formation of Mg on $\mathrm{Ag}(100)$. Applied Surface Science 1999, 151, (1-2), 40-48.

77. I. Campillo, V. M. S., J. M. Pitarke, E. V. Chulkov, A. Rubio, P. M. Echenique, Firstprinciples calculations of hot-electron lifetimes in metals. Phyiscal Review B 2001. 